\title{
Article
}

\section{Ring-theoretic (in)finiteness in reduced products of Banach algebras}

Daws, Matthew and Horvath, Bence

Available at https://clok.uclan.ac.uk/33955/

Daws, Matthew orcid iconORCID: 0000-0003-1707-4308 and Horvath, Bence (2020) Ring-theoretic (in)finiteness in reduced products of Banach algebras. Canadian Journal of Mathematics . ISSN 0008-414X

It is advisable to refer to the publisher's version if you intend to cite from the work. http://dx.doi.org/10.4153/S0008414X20000565

For more information about UCLan's research in this area go to http://www.uclan.ac.uk/researchgroups/ and search for < name of research Group>.

For information about Research generally at UCLan please go to http://www.uclan.ac.uk/research/

All outputs in CLoK are protected by Intellectual Property Rights law, including Copyright law. Copyright, IPR and Moral Rights for the works on this site are retained by the individual authors and/or other copyright owners. Terms and conditions for use of this material are defined in the policies page.

\section{CLoK}

Central Lancashire online Knowledge www.clok.uclan.ac.uk 


\title{
Ring-theoretic (in)finiteness in reduced products of Banach algebras
}

\author{
Matthew Daws and Bence Horváth
}

\begin{abstract}
We study ring-theoretic (in)finiteness properties - such as Dedekind-finiteness and proper infiniteness - of ultraproducts (and more generally, reduced products) of Banach algebras.

Whilst we characterise when an ultraproduct has these ring-theoretic properties in terms of its underlying sequence of algebras, we find that, contrary to the $C^{*}$-algebraic setting, it is not true in general that an ultraproduct has a ring-theoretic finiteness property if and only if "ultrafilter many" of the underlying sequence of algebras have the same property. This might appear to violate the continuous model theoretic counterpart of Łoś's Theorem; the reason it does not is that for a general Banach algebra, the ring theoretic properties we consider cannot be verified by considering a bounded subset of the algebra of fixed bound. For Banach algebras, we construct counter-examples to show, for example, that each component Banach algebra can fail to be Dedekind-finite while the ultraproduct is Dedekind-finite, and we explain why such a counter-example is not possible for $C^{*}$-algebras. Finally the related notion of having stable rank one is also studied for ultraproducts.
\end{abstract}

\section{Introduction}

The notion of central sequences (with respect to a limit, or an ultrafilter limit) has long been a key tool in the study and classification of von Neumann algebras (see [27, Section 3, Chapter XIV] as a starting point). More recently, such ideas have also become central to the classification of $C^{*}$-algebras, see [19] for example. The study of ultrapowers is intimately connected to model theory, and indeed continuous model theory has recently been successfully applied to the study of von Neumann and $C^{*}$-algebras, $[10,11]$. Furthermore, the analogue of the ultrapower where usual convergence is used, the asymptotic sequence algebra, appears in the study of the set theory of $C^{*}$-algebras, [9].

In constructing ultraproducts or the asymptotic sequence algebra, we of course only consider bounded sequences. Many properties of $C^{*}$-algebras, such as those considered in this paper - being Dedekind-finite, being properly infinite - can be verified for a $C^{*}$ algebra by only looking at bounded, in fact, norm one, elements. In this paper, we show that this is not true for general Banach algebras, and that furthermore, this has implications for ultraproducts, and asymptotic sequence algebras, of Banach algebras. For example, the property of being Dedekind-finite passes from each component Banach algebra to the asymptotic sequence algebra, but the converse is not true. This is a manifestation of the fact that the very language of continuous model theory involves the use of bounded metric spaces, [1]. We remark that the asymptotic sequence algebra appears

AMS subject classification: 46M07, 46H99 (primary); 16B99, 43A20 (secondary).

Keywords: Asymptotic sequence algebra, Banach algebra, bicyclic monoid, Cuntz semigroup, Dedekind-finite, Łoś's Theorem, properly infinite, stable rank one, semigroup algebra, ultraproduct. 
to have not been systematically studied for Banach algebras; we think that this is likely to prove to be a useful construction in general Banach algebra theory.

Let us now be more precise. The main objects of study in this paper are the following. Let $\left(\mathcal{A}_{n}\right)$ be a sequence of Banach algebras and let $\ell^{\infty}\left(\mathcal{A}_{n}\right)$ be the Banach space of all bounded sequences $\left(a_{n}\right)$, where $a_{n} \in \mathcal{A}_{n}$ for each $n$, turned into a Banach algebra with pointwise operations. Similarly, let $c_{0}\left(\mathcal{A}_{n}\right)$ be the subspace of sequences $\left(a_{n}\right)$ with $\lim _{n}\left\|a_{n}\right\|=0$. Then $c_{0}\left(\mathcal{A}_{n}\right)$ is a closed, two-sided ideal of $\ell^{\infty}\left(\mathcal{A}_{n}\right)$ and in fact, when each $\mathcal{A}_{n}$ is unital, $\ell^{\infty}\left(\mathcal{A}_{n}\right)$ is the multiplier algebra of $c_{0}\left(\mathcal{A}_{n}\right)$ (compare [9, Section 13] for example). The asymptotic sequence algebra $\operatorname{Asy}\left(\mathcal{A}_{n}\right)$ is the quotient algebra $\ell^{\infty}\left(\mathcal{A}_{n}\right) / c_{0}\left(\mathcal{A}_{n}\right)$. Let $\mathcal{U}$ be a non-principal ultrafilter on $\mathbb{N}$ and let $c \mathcal{U}\left(\mathcal{A}_{n}\right)$ be the closed, two-sided ideal of $\ell^{\infty}\left(\mathcal{A}_{n}\right)$ formed of sequences $\left(a_{n}\right)$ with $\lim _{n \rightarrow \mathcal{U}}\left\|a_{n}\right\|=$ 0 . The quotient $\ell^{\infty}\left(\mathcal{A}_{n}\right) / c_{\mathcal{U}}\left(\mathcal{A}_{n}\right)$ is the ultraproduct $\left(\mathcal{A}_{n}\right)_{\mathcal{U}}$, see [15]. As $c_{0}\left(\mathcal{A}_{n}\right) \subseteq$ $c_{\mathcal{U}}\left(\mathcal{A}_{n}\right)$ the ultraproduct is "smaller" than the asymptotic sequence algebra, although for the questions we consider here there will be little difference. If $\mathcal{A}_{n}=\mathcal{A}$ for all $n$, we write $\operatorname{Asy}(\mathcal{A})$ and $(\mathcal{A}) \mathcal{U}$, the latter known as the ultrapower of $\mathcal{A}$.

We shall denote by a capital letter $A$, and so forth, an element $A=\left(a_{n}\right) \in \ell^{\infty}\left(\mathcal{A}_{n}\right)$. Let $\pi: \ell^{\infty}\left(\mathcal{A}_{n}\right) \rightarrow \operatorname{Asy}\left(\mathcal{A}_{n}\right)$ and $\pi_{\mathcal{U}}: \ell^{\infty}\left(\mathcal{A}_{n}\right) \rightarrow\left(\mathcal{A}_{n}\right) \mathcal{U}$ be the quotient maps; then

$$
\|\pi(A)\|=\limsup _{n \rightarrow \infty}\left\|a_{n}\right\|, \quad\left\|\pi_{\mathcal{U}}(A)\right\|=\lim _{n \rightarrow \mathcal{U}}\left\|a_{n}\right\| .
$$

In particular, given any $a \in \operatorname{Asy}\left(\mathcal{A}_{n}\right)$ we can always find $A=\left(a_{n}\right) \in \ell^{\infty}\left(\mathcal{A}_{n}\right)$ with $\pi(A)=a$ and $\|A\|=\sup _{n}\left\|a_{n}\right\|=\|a\|$, and similarly for $\left(\mathcal{A}_{n}\right) \mathcal{U}$. As our notation indicates, we only work with sequences of algebras $\left(\mathcal{A}_{n}\right)$, and not with general nets, though our results could be formulated in a more general setting. We always assume, then, that our ultrafilters are non-principal, which on a countable indexing set, is equivalent to being countably-incomplete, [15, Section 1].

An approach we could have taken to our overall presentation would have been to work with "reduced products", compare [14, Section 2.3]. If $\mathcal{F}$ is merely a filter on $\mathbb{N}$ (and not necessarily an ultrafilter) we may still define $c_{\mathcal{F}}\left(\mathcal{A}_{n}\right)$ to be those sequences $\left(a_{n}\right) \in \ell^{\infty}\left(\mathcal{A}_{n}\right)$ such, for each $\epsilon>0$, that $\left\{n \in \mathbb{N}:\left\|a_{n}\right\|<\epsilon\right\} \in \mathcal{F}$. Then $c_{\mathcal{F}}\left(\mathcal{A}_{n}\right)$ is a closed, two-sided ideal, and so we may define $\left(\mathcal{A}_{n}\right)_{\mathcal{F}}=\ell^{\infty}\left(\mathcal{A}_{n}\right) / c_{\mathcal{F}}\left(\mathcal{A}_{n}\right)$. This definition agrees with the previous one if $\mathcal{F}$ does happen to be an ultrafilter. Furthermore, if $\mathcal{F}$ is the Fréchet filter (so $A \in \mathcal{F}$ if and only if $\mathbb{N} \backslash A$ is finite) then $c_{\mathcal{F}}\left(\mathcal{A}_{n}\right)=c_{0}\left(\mathcal{A}_{n}\right)$ and so $\left(\mathcal{A}_{n}\right)_{\mathcal{F}}=\operatorname{Asy}\left(\mathcal{A}_{n}\right)$. Consequently, we could have structured all our statements and proofs to be about reduced products. Instead, we felt that writing statements and proofs for $\operatorname{Asy}\left(\mathcal{A}_{n}\right)$ improved the readability (as we can work with "normal" convergence, and limits at $\infty$ ). Once the structure of an argument is understood, it is then easy to adapt it to work for (ultra)filter products.

As motivation, and to make explicit links with continuous logic, let us consider when $\operatorname{Asy}\left(\mathcal{A}_{n}\right)$ or $\left(\mathcal{A}_{n}\right) \mathcal{U}$ is unital. The first author considered the ultrapower case in $[5$, Proposition 2.1], showing that $(\mathcal{A})_{\mathcal{U}}$ is unital if and only if $\mathcal{A}$ is, the proof using just techniques from functional analysis. In Section 2 below, we consider how to use the tools of continuous model theory to show, and improve upon, this result. From this perspective, the bulk of the proof of [5, Proposition 2.1] is taken up with showing that one can write a sentence $\varphi$ in the language of Banach algebras such that a Banach algebra $\mathcal{A}$ is unital if and only if the interpretation $\varphi^{\mathcal{A}}$ of $\varphi$ in $\mathcal{A}$ is zero. This is not entirely 
trivial, as in continuous logic the existential and universal quantifiers are replaced by supremum and infimum. Once such a sentence has been found, Łośs Theorem for continuous model theory shows that $\varphi^{\mathcal{A}}=0$ if and only if $\varphi^{(\mathcal{A})_{\mathcal{U}}}=0$, hence immediately showing the result. In Section 2 below we show how to also obtain an analogous result for ultraproducts.

An interesting aspect of continuous logic is that the language of Banach algebras requires us to choose bounded domains for any sentence or term we use. These domains are typically chosen so that in an interpretation of the language, they are closed balls of varying radii. For the statement " $\mathcal{A}$ is unital" this seems innocuous, as units always have norm one. However, for an abstract Banach algebra (as opposed to $C^{*}$-algebras, or most concretely occurring Banach algebras) this is merely convention. Indeed, if we allow Banach algebras to have units of norm greater than one, then we can find a sequence $\left(\mathcal{A}_{n}\right)$ of unital Banach algebras such that $\left(\mathcal{A}_{n}\right)_{\mathcal{U}}$ is not unital, see Proposition 3.15. We shall explore this phenomena extensively for the algebraic properties we consider below.

Once units have been studied, it is natural to look at idempotents, that is, elements $p \in \mathcal{A}$ with $p^{2}=p$. Two idempotents $p, q$ are equivalent, written $p \sim q$, when there are $a, b \in \mathcal{A}$ with $p=a b$ and $q=b a$. This is indeed an equivalence relation, see Section 2 for this. We say that $p, q$ are orthogonal if $p q=0$ and $q p=0$. When $\mathcal{A}$ is a $C^{*}$-algebra, it is more natural to take account of the star-structure, and to ask that our idempotents are self-adjoint, giving the notion of a projection. In Section 2 we give a quick survey of the relation between idempotents and projections, showing in particular that for $C^{*}$-algebras, for the properties we consider, one can equivalently work with either idempotents, or projections.

A unital algebra $\mathcal{A}$ is properly infinite if there are orthogonal idempotents $p, q \in \mathcal{A}$ with $p \sim 1$ and $q \sim 1$. $\mathcal{A}$ is Dedekind-finite if $p \sim 1$ implies $p=1$, and is otherwise Dedekind-infinite. In this paper, we study how these notions interact with the ultraproduct, and, especially, the asymptotic sequence algebra constructions.

In Section 3, we show that if $\left(\mathcal{A}_{n}\right)$ is a sequence of Dedekind-finite Banach algebras then also $\operatorname{Asy}\left(\mathcal{A}_{n}\right)$ is Dedekind-finite. The converse is not, in general, true, but we show that it is under further conditions. The definition of $p \sim 1$ entails the existence of $a, b \in \mathcal{A}$ with $p=a b, b a=1$. To write a sentence in the language of Banach algebras expressing this requires us, amongst other things, to give a bounded domain to work in: that is, to norm bound $a, b$. For technical reasons, it is easier to work with the notion of being Dedekind-infinite, and we introduce two constants, $C_{\mathrm{DI}}(\mathcal{A})$ and $C_{\mathrm{DI}}^{\prime}(\mathcal{A})$, which measure how large $\|a\|\|b\|$, or $\|p\|$ where $p$ is idempotent, need to be to show that $\mathcal{A}$ is Dedekind-infinite; see Section 3.2 for precise statements.

We show in Proposition 3.4 that if $\left(\mathcal{A}_{n}\right)$ is a sequence of Dedekind-infinite Banach algebras, but with unbounded $C_{\mathrm{DI}}$ constants, then $\operatorname{Asy}\left(\mathcal{A}_{n}\right)$ is Dedekind-finite. We then show, by way of examples of weighted semigroup algebras, that such a sequence can exist. By contrast, such an example cannot occur for $C^{*}$-algebras, see Corollary 3.9.

This exploration also raises the question of renormings. For a unital Banach algebra, we can renorm so as to ensure the unit has norm 1 . We find examples which show that $C_{\mathrm{DI}}(\mathcal{A})$ and $C_{\mathrm{DI}}^{\prime}(\mathcal{A})$ need not be comparable, meaning that we can show $\mathcal{A}$ is Dedekind infinite for an idempotent $p$ of small norm, but to verify that $p \sim 1$, that is, find $a, b \in \mathcal{A}$ with $p=a b, 1=b a$, we need to use $a, b$ of large norm. We make some remarks on 
whether it is possible to renorm $\mathcal{A}$ to make $C_{\mathrm{DI}}(\mathcal{A})$ small, but ultimately leave this as an open question.

In Section 4, we perform a similar analysis for being properly infinite: if $\operatorname{Asy}\left(\mathcal{A}_{n}\right)$ is properly infinite then $\mathcal{A}_{n}$ is properly infinite for large enough $n$. Again, the converse holds when we have uniform norm control on the norms of the elements of $\mathcal{A}$ showing that the idempotents $p, q$, involved in the definition of "properly infinite", are equivalent to 1 . A more involved example of a weighted semigroup algebra provides a counterexample, Theorem 4.11. We again show that it is possible to control the norms of the idempotents without being able to control the norms of elements involved in verifying that $p \sim 1, q \sim 1$, see Proposition 4.13. We also investigate renorming questions. When we do have sufficient norm control, we completely characterise when $\operatorname{Asy}\left(\mathcal{A}_{n}\right)$ is properly infinite, Proposition 4.17. This is again achieved by introducing constants $C_{\mathrm{PI}}^{\prime}(\mathcal{A})$ and $C_{\mathrm{PI}}(\mathcal{A})$, which measure how big the product of the norms $\|p\|\|q\|$ are which witness proper infiniteness, and respectively, the product of the norms of the elements implementing the equivalence of the idempotents. Along the way, we show that there is an isometric embedding of the inductive limit of Banach algebras $\left(\mathcal{A}_{n}\right)$ into $\operatorname{Asy}\left(\mathcal{A}_{n}\right)$, and use this to draw conclusions about when the inductive limit is properly infinite.

Finally, in Section 5, we consider the property of having stable rank one, which we view as a strengthening of being Dedekind-finite. Here $\operatorname{Asy}(\mathcal{A})$ having stable rank one implies the same for $\mathcal{A}$; again the converse does not hold, which we show by way of a counter-example. Two themes running through our consideration of all three properties are "lifting" properties from $\operatorname{Asy}\left(\mathcal{A}_{n}\right)$ to $\ell^{\infty}\left(\mathcal{A}_{n}\right)$, which we view as being interesting from a technical viewpoint; and also the certain "norm control" considerations mentioned above.

The organisation of the paper is as follows. In Section 2 below, we first consider some aspects of continuous model theory, and in particular Łoś's Theorem, before giving some background results about idempotents, projections, and present some results about being "close" to an idempotent. In the subsequent sections we study, respectively, being Dedekind-finite, being properly infinite, and having stable rank one. We finish the paper with some open problems.

\section{Preliminaries}

For us, a Banach algebra $\mathcal{A}$ will always have a contractive product $\|a b\| \leq\|a\|\|b\|$. If $\mathcal{A}$ is unital then we assume that the unit 1 has $\|1\|=1$. These assumptions can always be achieved by giving $\mathcal{A}$ an equivalent norm (see e.g. [3, Proposition 2.1.9] and the comment following it). As many of the results in this paper depend upon exact norm control, and not just upon the equivalence class of the norm, we should be a little careful of renorming arguments. The reader is pointed to Section 3.4 and Propositions 4.15 and 4.16 below for a wider discussion.

Let us make some remarks about continuous logic. Again, we shall motivate this discussion by considering when $\mathcal{A}$ is unital. That $\mathcal{A}$ is unital, with unit $e \in \mathcal{A}$, can be expressed in first-order logic as $\exists e \in \mathcal{A}, \forall a \in \mathcal{A}$, ae $=e a=a$. However, in continuous logic, we have to use sup and inf in place of $\forall$ and $\exists$, and furthermore, we can only quantify over bounded subsets of $\mathcal{A}$. In fact, typically we define bounded domains $B_{n}$ which, when interpreted in $\mathcal{A}$ will be the closed ball of radius $n \in \mathbb{N}$. Consider the 
sentence

$$
\varphi=\inf _{e \in B_{1}} \sup _{a \in B_{1}} \max (\|a e-a\|,\|e a-a\|)
$$

Arguing exactly as in the proof of [5, Proposition 2.1], we can show that a Banach algebra $\mathcal{A}$ is unital if and only if $\varphi^{\mathcal{A}}=0$, that is, the interpretation of $\varphi$ in $\mathcal{A}$ is zero.

Łoś's Theorem for continuous model theory, [11, Proposition 4.3] or [1, Theorem 5.4], shows that $\mathcal{A}$ will satisfy this sentence if and only if any ultrapower $(\mathcal{A}) \mathcal{U}$ does. This immediately shows [5, Proposition 2.1]. In effect, Łoś's Theorem takes care of the "ultraproduct bookkeeping" for us. In fact, we can use Łośs Theorem to improve [5, Proposition 2.1], as follows.

Proposition 2.1 Let $\left(\mathcal{A}_{n}\right)$ be a sequence of Banach algebras. Then $\left(\mathcal{A}_{n}\right)_{\mathcal{U}}$ is unital if and only if there is $U \in \mathcal{U}$ with $\mathcal{A}_{n}$ unital for each $n \in U$.

Proof Set $\mathcal{B}=(\mathcal{A})_{\mathcal{U}}$ and suppose that $\mathcal{B}$ is unital. By Łoś's Theorem we know that $\varphi^{\mathcal{B}}=\lim _{n \rightarrow \mathcal{U}} \varphi^{\mathcal{A}_{n}}$. We are supposing that $\varphi^{\mathcal{B}}=0$, so for $\epsilon \in(0,1 / 4)$ to be chosen later, there is $U \in \mathcal{U}$ with $\varphi^{\mathcal{A}_{n}}<\epsilon$ for $n \in U$. Given such an $n$ we can find $e \in \mathcal{A}_{n}$ with $\|e\| \leq 1$ and $\|e a-a\|,\|a e-a\| \leq \epsilon\|a\|$ for each $a \in \mathcal{A}_{n}$. We now apply Corollary 2.7 below, to find $p \in \mathcal{A}_{n}$ with $p^{2}=p$ and $\|e-p\| \leq f_{1}(\epsilon)$, where $f_{1}$ is defined by equation 2.1 below.

Then $\|p c-c\| \leq\|p-e\|\|c\|+\epsilon\|c\| \leq\left(f_{1}(\epsilon)+\epsilon\right)\|c\|$ for each $c \in \mathcal{A}_{n}$. If we choose $\epsilon>0$ small enough then $f_{1}(\epsilon)+\epsilon<1$. Given $a \in \mathcal{A}_{n}$ let $b=a-p a$ so that $p b=0$ because $p^{2}=p$. If $b \neq 0$ then we conclude that $\|b\|=\|p b-b\|<\|b\|$, a contradiction. Hence $p a=a$ and similarly $a p=a$, and so $p$ is the unit of $\mathcal{A}_{n}$, as required.

The converse is clear.

We shall show later in Proposition 3.15 that if we allow a Banach algebra to have a unit of norm greater than 1 then the previous proposition is false.

Let us make two remarks about this treatment of $\mathcal{A}$ being unital. The first remark is that we could work with the bounded domain $B_{1}$ above only because of our assumption that if a Banach algebra is unital, then its unit has norm 1. However, this is only ensured by a renorming argument. By contrast, for a $C^{*}$-algebra, a unit $e$ is self-adjoint (because units are unique) and an idempotent, and so is a projection, and hence has norm 1 automatically.

Renorming arguments are not problematic when considering ultrapowers (or $\operatorname{Asy}(\mathcal{A})$ ) but can become questionable when considering ultraproducts (or $\operatorname{Asy}\left(\mathcal{A}_{n}\right)$ for a varying sequence $\left(\mathcal{A}_{n}\right)$ ). We shall make further remarks later, see Section 3.4 below.

The second remark is that the bulk of the argument of, for example, Proposition 2.1 is functional analytic: showing that if $\varphi^{\mathcal{A}}$ is small, then in fact $\mathcal{A}$ is unital. The necessary Banach algebraic techniques for the properties we consider below are only more involved, and for this reason, we have chosen to present our results in a way which does not explicitly use continuous model theory. 


\subsection{Background results about idempotents}

Let us quickly recall why $\sim$ is an equivalence relation on idempotents. Only transitivity is non-trivial. Let $p \sim q$ and $q \sim r$, say with $p=a b, q=b a$ and $q=c d, r=d c$. Then $p=p^{2}=a b a b=a q b=(a c)(d b)$ and $(d b)(a c)=d q c=d c d c=r^{2}=r$ so $p \sim r$.

For a $C^{*}$-algebra $\mathcal{A}$, rather than considering idempotents, it is usual to consider projections, which are by definition self-adjoint idempotents: $p \in \mathcal{A}$ with $p=p^{*}=p^{2}$. It is a fun exercise to show that an idempotent in a $C^{*}$-algebra is a projection if and only if it has norm one. Further, the natural equivalence of projections is that of Murray-von Neumann equivalence, which says that $p \approx q$ if and only if $p=v^{*} v, q=v v^{*}$ for some $v \in \mathcal{A}$ (which is necessarily a partial isometry). The proof in the previous paragraph still works to show that $\approx$ is an equivalence relation. Finally, it is then usual to define properly infinite and Dedekind-(in)finite using projections and Murray-von Neumann equivalence.

We claim that it does not matter, for a $C^{*}$-algebra, if we use our definitions or the $C^{*}$-definitions. This is folklore, but we have been unable to find a reference, so to aid the reader, we give the argument. For the next few results, we fix a unital $C^{*}$-algebra $\mathcal{A}$.

Lemma 2.2 Let $p \in \mathcal{A}$ be an idempotent. There is a projection $q \in \mathcal{A}$ with $p \sim q$. We can arrange for $p q=q, q p=p$ or for $p q=p, q p=q$.

Proof This is [26, Exercise 3.11] or [3, Proposition 3.2.10]. Set $a=1+\left(p-p^{*}\right)^{*}(p-$ $\left.p^{*}\right) \geq 1$ so $a$ is invertible. Then $p a=p p^{*} p=a p$ and also $p^{*} a=a p^{*}$. Set $q=p p^{*} a^{-1}=$ $a^{-1} p p^{*}$. Then $q^{2}=a^{-1} p p^{*} p p^{*} a^{-1}=a^{-1} a p p^{*} a^{-1}=q$ and $q=q^{*}$. Also $p q=q$ while $q p=a^{-1} p p^{*} p=a^{-1} a p=p$, hence $p \sim q$. If instead we set $r=p^{*} p a^{-1}=a^{-1} p^{*} p$ then $r^{2}=r=r^{*}$ while $p r=p$ and $r p=r$.

Lemma 2.3 Let $p, q \in \mathcal{A}$ be projections with $p \sim q$. Then $p \approx q$.

Proof This is [3, Proposition 3.2.10]. Suppose $p=a b$ and $q=b a$. Let $c=q b p$ thus $q c=c=c p$; as also $c=b a b a b$ we have that $a c=p$ and $c a=q$, and consequently $c a c=c p=c$.

Suppose $p \neq 0$. We have that $p=p^{*} p=c^{*} a^{*} a c \leq\|a\|^{2} c^{*} c$. Working in $p \mathcal{A} p$, we see that $c^{*} c$ is invertible, so there is $d \in p \mathcal{A l} p$ with $d|c|=|c| d=p$. Set $u=c d$ so $u^{*} u=d^{*}|c|^{2} d=p$. Then $q u=c a c d=c d=u$ and hence $u u^{*}=u u^{*} q=c d d^{*} c^{*} c a=$ $c d d^{*}|c|^{2} a=c d p|c| a=c d|c| a=c p a=c a c a=q$. Thus $p \approx q$.

If $p=0, q \neq 0$ then swap the roles of $p$ and $q$. If $p=q=0$ then clearly $p \approx q$.

Proposition 2.4 $\mathcal{A}$ is properly infinite as a Banach algebra if and only if it is properly infinite as a $C^{*}$-algebra.

Proof If $\mathcal{A}$ is properly infinite as a Banach algebra then there are idempotents $p, q$ with $p q=q p=0$ and $p \sim 1, q \sim 1$. By Lemma 2.2 there are projections $p^{\prime}, q^{\prime}$ with $p p^{\prime}=p^{\prime}, p^{\prime} p=p$ and $q q^{\prime}=q, q^{\prime} q=q^{\prime}$. Then $1 \sim p \sim p^{\prime}$ so $p^{\prime} \approx 1$ by Lemma 2.3 , and similarly $q^{\prime} \approx 1$. Also $q^{\prime} p^{\prime}=q^{\prime} q p p^{\prime}=0$ and similarly $p^{\prime} q^{\prime}=0$, hence $\mathcal{A}$ is properly infinite as a $C^{*}$-algebra. The converse is clear. 
Proposition 2.5 $\mathcal{A}$ is Dedekind-finite as a Banach algebra if and only if it is Dedekind-finite as a $C^{*}$-algebra.

Proof If $\mathcal{A}$ is Dedekind-finite as a $C^{*}$-algebra, then let $p \in \mathcal{A}$ be an idempotent with $p \sim 1$. There is a projection $q \in \mathcal{A}$ with $q \sim p$. Thus $q \sim p \sim 1$ so $q \sim 1$ hence $q \approx 1$, and consequently $q=1$. We can arrange that $p q=q$, so as $q=1$, we conclude that $1=q=p q=p$. Therefore $\mathcal{A}$ is Dedekind-finite as a Banach algebra. The converse is clear.

We now return to the general case. For $M>0$ define

$$
f_{M}:[0,1 / 4) \rightarrow \mathbb{R} ; \quad f_{M}(t)=(M+1 / 2)\left((1-4 t)^{-1 / 2}-1\right)
$$

It is clear that $f_{M}$ is a non-negative continuous function. Furthermore, $f_{M} \leq f_{N}$ when $N>M>0$.

The following lemma is well-known, it can be found for example in [18, Lemma 2.1] without a proof. For completeness we provide a (functional calculus argument based) proof. In what follows, if $\mathcal{A}$ is an algebra, then $\operatorname{inv}(\mathcal{A})$ denotes the group of invertible elements of $\mathcal{A}$. If $a, b \in \mathcal{A}$, then the commutator of $a$ and $b$ is $[a, b]:=a b-b a$.

Proposition 2.6 Let $\mathcal{A}$ be a unital Banach algebra, and let $a \in \mathcal{A}$ be such that $v:=\left\|a^{2}-a\right\|<1 / 4$. Then there is an idempotent $p \in \mathcal{A}$ such that $\|p-a\| \leq f_{\|a\|}(v)$ holds. Moreover, if $y \in \mathcal{A}$ is such that $[y, a]=0$ then $[y, p]=0$.

Proof As $v<1 / 4$, it follows that the series $\sum_{n=0}^{\infty}\left(\begin{array}{c}2 n \\ n\end{array}\right) v^{n}$ converges in $[0, \infty)$ with sum $(1-4 v)^{-1 / 2}$, consequently

$$
s:=\sum_{n=0}^{\infty}\left(\begin{array}{c}
2 n \\
n
\end{array}\right)\left(a-a^{2}\right)^{n}
$$

is absolutely convergent and therefore convergent in $\mathcal{A}$. Let us define $p:=(a-1 / 2) s+1 / 2$. Clearly, if $y \in \mathcal{A}$ is such that $[y, a]=0$ then $[y, s]=0$, and consequently $[y, p]=0$. We show that $p \in \mathcal{A}$ is an idempotent, which is equivalent to showing that $(2 p-1)^{2}=1$.

We first observe that by the Cauchy product formula

$$
s^{2}=\sum_{n=0}^{\infty}\left(\sum_{k=0}^{n}\left(\begin{array}{c}
2 k \\
k
\end{array}\right)\left(\begin{array}{c}
2(n-k) \\
n-k
\end{array}\right)\right)\left(a-a^{2}\right)^{n}=\sum_{n=0}^{\infty} 4^{n}\left(a-a^{2}\right)^{n} .
$$

Secondly, by $v<1 / 4$ it follows that $1-4 a+4 a^{2} \in \operatorname{inv}(\mathcal{A})$ with $\left(1-4 a+4 a^{2}\right)^{-1}=$ $\sum_{n=0}^{\infty}\left(4\left(a-a^{2}\right)\right)^{n}$ by the Carl Neumann series. Thus $s^{2}=\left(1-4 a+4 a^{2}\right)^{-1}$ and consequently $(2 p-1)^{2}=((2 a-1) s)^{2}=(2 a-1)^{2} s^{2}=\left(4 a^{2}-4 a+1\right)\left(1-4 a+4 a^{2}\right)^{-1}=1$. 
Moreover, we have that

$$
\begin{aligned}
\|p-a\| & =\|(a-1 / 2) s+1 / 2-a\|=\|(a-1 / 2)(s-1)\| \\
& \leq(\|a\|+1 / 2)\|s-1\| \leq(\|a\|+1 / 2) \sum_{n=1}^{\infty}\left(\begin{array}{c}
2 n \\
n
\end{array}\right)\left\|a-a^{2}\right\|^{n} \\
& =(\|a\|+1 / 2)\left((1-4 v)^{-1 / 2}-1\right)=f_{\|a\|}(v)
\end{aligned}
$$

by the definition of $f_{\|a\|}$.

Corollary 2.7 Let $\mathcal{A}$ be a Banach algebra, and let $a \in \mathcal{A}$ with $v:=\left\|a^{2}-a\right\|<1 / 4$ and $f_{\|a\|}(v)<1$. Then there is an idempotent $p \in \mathcal{A}$ with $\|p-a\| \leq f_{\|a\|}(v)$.

Proof If $\mathcal{A}$ is not unital, consider the unitisation $\mathcal{A}^{\sharp}$ with adjoined unit 1 . So $\mathcal{A}^{\sharp}=$ $\mathcal{A} \oplus \mathbb{C} 1$ with norm $\|a+\alpha 1\|=\|a\|+|\alpha|$. By the preceding applied to $\mathcal{A}^{\sharp}$ there is $p=q+\alpha 1$ with $p^{2}=p$ and $\|p-a\| \leq f_{\|a\|}(v)$. Thus $p^{2}=q^{2}+2 \alpha q+\alpha^{2} 1$ so $p^{2}=p$ implies that either $\alpha=0$ or $\alpha=1$. If $\alpha=1$ then $\|p-a\|=\|q-a\|+|\alpha| \geq 1$ a contradiction. So $\alpha=0$ and $p=q$ is an idempotent.

A related result is the following, which is also folklore, a stronger version of which was proved by Zemánek in [28, Lemma 3.1]. For the convenience of the reader we give a self-contained elementary proof.

Lemma 2.8 Let $\mathcal{A}$ be a unital Banach algebra, and let $p, q \in \mathcal{A}$ be idempotents with $\| p-$ $q \|<1$. Then $p \sim q$.

Proof We first observe that $(p-q)^{2}$ commutes with $p$ and $q$. Indeed,

$$
\begin{aligned}
p(p-q)^{2} & =p(p-p q-q p+q)=p-p q p \\
& =(p-p q-q p+q) p=(p-q)^{2} p,
\end{aligned}
$$

and similarly for $q$ and $(p-q)^{2}$. Now since $\|p-q\|<1$, clearly $\left\|(p-q)^{2}\right\|<1$ and thus as in the proof of Proposition 2.6, the series

$$
d:=\sum_{n=0}^{\infty}\left(\begin{array}{c}
2 n \\
n
\end{array}\right) \frac{(p-q)^{2 n}}{4^{n}}
$$

converges (absolutely) in $\mathcal{A}$, and $d$ commutes with $p$ and $q$.

Again, as in the proof of Proposition 2.6 we conclude $d^{2}=\left(1-(p-q)^{2}\right)^{-1}$. Another easy calculation shows

$$
\begin{aligned}
(p+q-1)^{2} & =p+p q-p+q p+q-q-p-q+1 \\
& =1-p+p q+q p-q=1-(p-q)^{2} .
\end{aligned}
$$

We define $c:=d(p+q-1)$. Since $p$ and $q$ commute with $d$, it follows that

$$
c^{2}=d^{2}(p+q-1)^{2}=d^{2}\left(1-(p-q)^{2}\right)=1 .
$$


We are now ready to show that $p \sim q$. To see this, we first observe

$$
\begin{aligned}
& p c=p d(p+q-1)=d p(p+q-1)=d(p+p q-p)=d p q \\
& c q=d(p+q-1) q=d(p q+q-q)=d p q
\end{aligned}
$$

and thus $p c=c q$. Consequently $(p c)(c p)=p c^{2} p=p$ and $(c p)(p c)=c p c=c^{2} q=q$ follow, concluding the proof.

Remark 2.9 From this proof, we see that $p=a b$ and $q=b a$ for $a, b \in \mathcal{A}$, where $a=p c, b=c p$, and $c=d(p+q-1)$, where $d$ is given by a power series which yields the norm estimate

$$
\|d\| \leq\left(1-\left\|(p-q)^{2}\right\|\right)^{-1 / 2} \leq\left(1-\|p-q\|^{2}\right)^{-1 / 2} .
$$

Thus

$$
\begin{aligned}
\|a\|\|b\| & \leq\|p\|^{2}\|p+q-1\|^{2}\left(1-\|p-q\|^{2}\right)^{-1} \\
& \leq\|p\|^{2}(\|2 p-1\|+\|p-q\|)^{2}\left(1-\|p-q\|^{2}\right)^{-1} .
\end{aligned}
$$

\section{Dedekind-finiteness}

In this section, we show that if $\mathcal{A}_{n}$ is Dedekind-finite for each $n$, then also $\operatorname{Asy}\left(\mathcal{A}_{n}\right)$ is Dedekind-finite. The converse is not true without some form of "norm control", and we provide a counter-example in the Banach algebra case, while also clarifying why the converse does hold for $C^{*}$-algebras.

\subsection{When the sequence consists of Dedekind-finite algebras}

In the following proof, for clarity, given a sequence $\left(\mathcal{A}_{n}\right)$ of unital algebras, we write $1_{n}$ for the unit of $\mathcal{A}_{n}$.

Theorem 3.1 Let $\left(\mathcal{A}_{n}\right)$ be a sequence of Dedekind-finite Banach algebras. Then $\operatorname{Asy}\left(\mathcal{A}_{n}\right)$ is Dedekind-finite.

Proof Let $p \in \operatorname{Asy}\left(\mathcal{A}_{n}\right)$ be an idempotent. Choose $X=\left(x_{n}\right) \in \ell^{\infty}\left(\mathcal{A}_{n}\right)$ with $\pi(X)=$ $p$, so that $\pi\left(X^{2}\right)=\pi(X)^{2}=p^{2}=p=\pi(X)$, or equivalently, $X-X^{2} \in c_{0}\left(\mathcal{A}_{n}\right)$. Let us introduce $v_{n}:=\left\|x_{n}-x_{n}^{2}\right\|$ for every $n \in \mathbb{N}$, then $\lim _{n \rightarrow \infty} v_{n}=0$. In particular, there is $N \in \mathbb{N}$ such that for every $n \geq N$ we have $v_{n}<1 / 8$. In view of Proposition 2.6, for every $n \geq N$ there is an idempotent $p_{n}^{\prime} \in \mathcal{A}_{n}$ with $\left\|x_{n}-p_{n}^{\prime}\right\| \leq f_{\left\|x_{n}\right\|}\left(v_{n}\right) \leq f_{\|X\|}\left(v_{n}\right) \leq$ $f_{\|X\|}(1 / 8)$. By continuity of $f_{\|X\|}$, it follows that $\lim _{n \geq N} f_{\|X\|}\left(v_{n}\right)=0$; consequently $\lim _{n \geq N}\left\|x_{n}-p_{n}^{\prime}\right\|=0$. For every $n \in \mathbb{N}$ we define

$$
p_{n}:= \begin{cases}p_{n}^{\prime} & \text { if } n \geq N \\ 0 & \text { otherwise }\end{cases}
$$

Since $\left\|p_{n}^{\prime}\right\| \leq\left\|p_{n}^{\prime}-x_{n}\right\|+\left\|x_{n}\right\| \leq f_{\|X\|}(1 / 8)+\|X\|$ for all $n \geq N$, it follows that $P:=$ $\left(p_{n}\right)$ is an idempotent in $\ell^{\infty}\left(\mathcal{A}_{n}\right)$. We observe that $p=\pi(P)$ by $\lim _{n \geq N}\left\|x_{n}-p_{n}^{\prime}\right\|=0$. 
Now suppose further that $p \sim 1$, so there exist $a, b \in \operatorname{Asy}\left(\mathcal{A}_{n}\right)$ such that $1=a b$ and $p=b a$. There are $A=\left(a_{n}\right), B=\left(b_{n}\right) \in \ell^{\infty}\left(\mathcal{A}_{n}\right)$ such that $a=\pi(A)$ and $b=\pi(B)$, consequently $\lim _{n \rightarrow \infty}\left\|1_{n}-a_{n} b_{n}\right\|=0$ and $\lim _{n \rightarrow \infty}\left\|p_{n}-b_{n} a_{n}\right\|=0$.

Now let $\delta \in(0,1)$ be such that

$$
\|A\|\|B\| \delta /(1-\delta)+2 \delta<1 .
$$

Let $M \geq N$ be such that for all $n \geq M$ the inequality $\left\|1_{n}-a_{n} b_{n}\right\|<\delta$ holds, then $u_{n}:=$ $a_{n} b_{n} \in \operatorname{inv}\left(\mathcal{A}_{n}\right)$ with $\left\|1_{n}-u_{n}^{-1}\right\|<\delta /(1-\delta)$. For every $n \geq M$, let $q_{n}:=b_{n} u_{n}^{-1} a_{n}$, then $q_{n} \in \mathcal{A}_{n}$ is an idempotent with $q_{n} \sim 1_{n}$. Since $\mathcal{A}_{n}$ is Dedekind-finite, it follows for all $n \geq M$ that $q_{n}=1_{n}$.

We need to show that $p=1$ holds, which is equivalent to showing $\lim _{n \rightarrow \infty} \| 1_{n}-$ $p_{n} \|=0$. Since $1_{n}-p_{n} \in \mathcal{A}_{n}$ is an idempotent for all $n \in \mathbb{N}$, it is enough to show that eventually $\left\|1_{n}-p_{n}\right\|<1$, compare Remark 3.3 below. Let $K \geq M$ be such that for every $n \geq K$

$$
\left\|x_{n}-b_{n} a_{n}\right\|<\delta, \quad\left\|x_{n}-p_{n}^{\prime}\right\|<\delta .
$$

Then for every $n \geq K$ we have $p_{n}=p_{n}^{\prime}$ and $1_{n}=q_{n}$, thus

$$
\begin{aligned}
\left\|1_{n}-p_{n}^{\prime}\right\| & =\left\|q_{n}-p_{n}^{\prime}\right\| \\
& =\left\|b_{n} u_{n}^{-1} a_{n}-p_{n}^{\prime}\right\| \\
& \leq\left\|b_{n} u_{n}^{-1} a_{n}-b_{n} a_{n}\right\|+\left\|b_{n} a_{n}-x_{n}\right\|+\left\|x_{n}-p_{n}^{\prime}\right\| \\
& \leq\left\|b_{n}\right\|\left\|u_{n}^{-1}-1_{n}\right\|\left\|a_{n}\right\|+\left\|b_{n} a_{n}-x_{n}\right\|+\left\|x_{n}-p_{n}^{\prime}\right\| \\
& \leq\|A\|\|B\| \delta /(1-\delta)+2 \delta \\
& <1 .
\end{aligned}
$$

This concludes the proof.

Remark 3.2 We note that the proof above gives some extra information. Indeed, the first paragraph of the proof of Theorem 3.1 shows precisely that idempotents from the asymptotic sequence algebra $\operatorname{Asy}\left(\mathcal{A}_{n}\right)$ can always be lifted to idempotents in $\ell^{\infty}\left(\mathcal{A}_{n}\right)$.

\subsection{When the asymptotic sequence algebra is Dedekind-finite.}

In this section we demonstrate that the converse of Theorem 3.1 holds for certain specific cases; but in general it does not hold, which we show by way of a counter-example.

In order to do this, let us introduce the following auxiliary quantity. For a unital Banach algebra $\mathcal{A}$, we define

$$
C_{\mathrm{DI}}(\mathcal{A}):=\inf \{\|a\|\|b\|: a, b \in \mathcal{A} \text { and } a b=1 \text { and } b a \neq 1\} .
$$

We may also introduce the auxiliary constant

$$
C_{\mathrm{DI}}^{\prime}(\mathcal{A}):=\inf \left\{\|p\|: p \in \mathcal{A}, p^{2}=p, p \sim 1 \text {, and } p \neq 1\right\} .
$$

If $\mathcal{A}$ is Dedekind-infinite then $1 \leq C_{\mathrm{DI}}(\mathcal{A})<+\infty$, otherwise (that is, if $\mathcal{A}$ is Dedekind-finite) $C_{\mathrm{DI}}(\mathcal{A})=+\infty$, and similarly for $C_{\mathrm{DI}}^{\prime}$. Clearly $C_{\mathrm{DI}}^{\prime}(\mathcal{A}) \leq C_{\mathrm{DI}}(\mathcal{A})$, 
but otherwise these quantities are not comparable, see Proposition 3.12. As a definition, perhaps $C_{\mathrm{DI}}^{\prime}(\mathcal{A})$ seems more natural, but we shall see that $C_{\mathrm{DI}}(\mathcal{A})$ is more useful in constructions.

Remark 3.3 If $p \in \mathcal{A}$ is an idempotent, then $p^{n}=p$ for any $n \in \mathbb{N}$, and so $\|p\| \geq 1$ or $p=0$. Suppose we have $a, b \in \mathcal{A}$ with $a b=1$. Then $p=b a$ is an idempotent, and hence so is $1-p$, hence either $p=1$ or $\|1-p\| \geq 1$. So in the definition of $C_{\mathrm{DI}}$ we also have that $b a$ is far away from 1 .

Proposition 3.4 Let $\left(\mathcal{A}_{n}\right)$ be a sequence of unital Banach algebras such that $C_{D I}\left(\mathcal{A}_{n}\right) \rightarrow$ $+\infty$. Then $\operatorname{Asy}\left(\mathcal{A}_{n}\right)$ is Dedekind-finite.

Proof Let $A=\left(a_{n}\right), B=\left(b_{n}\right) \in \ell^{\infty}\left(\mathcal{A}_{n}\right)$ be such that $\pi(A) \pi(B)=1$. We wish to prove that $\pi(B) \pi(A)=1$. By the assumption we can take $N^{\prime} \in \mathbb{N}$ such that $C_{\mathrm{DI}}\left(\mathcal{A}_{n}\right)>$ $2\|A\|\|B\|+1$ whenever $n \geq N^{\prime}$. Let us define $u_{n}:=a_{n} b_{n}$ for every $n \in \mathbb{N}$. Since $\lim _{n \rightarrow \infty}\left\|1_{n}-u_{n}\right\|=0$, we can pick $N \geq N^{\prime}$ such that $\left\|1_{n}-u_{n}\right\|<(2\|A\|\|B\|+1)^{-1}$ for all $n \geq N$. Then $u_{n} \in \operatorname{inv}\left(\mathcal{A}_{n}\right)$ and $\left\|1_{n}-u_{n}^{-1}\right\| \leq(2\|A\|\|B\|)^{-1}$ and $\left\|u_{n}^{-1}\right\| \leq$ $(2\|A\|\|B\|+1)(2\|A\|\|B\|)^{-1}$ for all $n \geq N$.

Let us define $q_{n}:=b_{n} u_{n}^{-1} a_{n}$ for every $n \geq N$. As $a_{n}\left(b_{n} u_{n}^{-1}\right)=1_{n}$ it follows that $q_{n} \in \mathcal{A}_{n}$ is an idempotent with $q_{n} \sim 1_{n}$. Clearly, either $q_{n}=1_{n}$ or $q_{n} \neq 1_{n}$. Assume towards a contradiction that there is some $m \geq N$ with $q_{m} \neq 1_{m}$. Then

$$
C_{\mathrm{DI}}\left(\mathcal{A}_{m}\right) \leq\left\|b_{m} u_{m}^{-1}\right\|\left\|a_{m}\right\| \leq\left\|b_{m}\right\|\left\|u_{m}^{-1}\right\|\left\|a_{m}\right\| \leq(2\|A\|\|B\|+1) / 2,
$$

which is impossible. Hence $q_{n}=1_{n}$ for all $n \geq N$.

From $\pi(A) \pi(B)=1$ it follows that $\pi(B) \pi(A) \in \operatorname{Asy}\left(\mathcal{A}_{n}\right)$ is an idempotent, which is equivalent to saying that $\lim _{n \rightarrow \infty}\left\|b_{n} a_{n} b_{n} a_{n}-b_{n} a_{n}\right\|=0$. Let $M^{\prime} \geq N$ be such that $v_{n}:=\left\|b_{n} a_{n} b_{n} a_{n}-b_{n} a_{n}\right\|<1 / 8$ whenever $n \geq M^{\prime}$. By Proposition 2.6 there is an idempotent $p_{n}^{\prime} \in \mathcal{A}_{n}$ such that $\left\|b_{n} a_{n}-p_{n}^{\prime}\right\| \leq f_{\|A\|\|B\|}\left(v_{n}\right)$, whenever $n \geq M^{\prime}$. Let $p_{n}:=p_{n}^{\prime}$ if $n \geq M^{\prime}$ and $p_{n}:=1_{n}$ if $n<M^{\prime}$. Then $P:=\left(p_{n}\right) \in \ell^{\infty}\left(\mathcal{A}_{n}\right)$ is an idempotent with $\pi(B) \pi(A)=\pi(P)$.

Let $M \geq M^{\prime}$ be such that $\left\|b_{n} a_{n}-p_{n}\right\|<1 / 2$ for all $n \geq M$. Thus we obtain

$$
\begin{aligned}
\left\|1_{n}-p_{n}\right\| & =\left\|q_{n}-p_{n}\right\| \leq\left\|q_{n}-b_{n} a_{n}\right\|+\left\|b_{n} a_{n}-p_{n}\right\| \\
& \leq\left\|b_{n}\right\|\left\|u_{n}^{-1}-1_{n}\right\|\left\|a_{n}\right\|+\left\|b_{n} a_{n}-p_{n}\right\| \\
& <\frac{\|A\|\|B\|}{2\|A\|\|B\|}+1 / 2 \\
& =1
\end{aligned}
$$

and hence, by Remark 3.3, $1_{n}=p_{n}$ for all $n \geq M$. This yields $\pi(B) \pi(A)=\pi(P)=\pi(1)$, showing that $\operatorname{Asy}\left(\mathcal{A}_{n}\right)$ is Dedekind-finite.

In particular as $C_{\mathrm{DI}}^{\prime}(\mathcal{A}) \leq C_{\mathrm{DI}}(\mathcal{A})$ and $C_{\mathrm{DI}}^{\prime}(\mathcal{A})<+\infty$ if and only if $C_{\mathrm{DI}}(\mathcal{A})<+\infty$, we immediately obtain the following.

Corollary 3.5 Let $\left(\mathcal{A}_{n}\right)$ be a sequence of unital Banach algebras such that $C_{D I}^{\prime}\left(\mathcal{A}_{n}\right) \rightarrow+\infty$. Then $\operatorname{Asy}\left(\mathcal{A}_{n}\right)$ is Dedekind-finite. 


\subsection{A counter-example}

We shall now construct Banach algebras which satisfy the hypothesises of Proposition 3.4 .

Let $I$ be a non-empty set. For a fixed $s \in I, \delta_{s}$ denotes the function

$$
\delta_{s}(t):= \begin{cases}1 & \text { if } t=s \\ 0 & \text { if } t \neq s .\end{cases}
$$

Let $v: I \rightarrow(0,+\infty)$ be a function. We define

$$
\ell^{1}(I, v):=\left\{f: I \rightarrow \mathbb{C}:\|f\|_{v}:=\sum_{s \in I}|f(s)| v(s)<+\infty\right\} .
$$

We have that $\ell^{1}(I, v)=\overline{\operatorname{span}\left\{\delta_{s}: s \in I\right\}}\|\cdot\|_{v}$, and further we can write $f=\sum_{s \in I} f(s) \delta_{s}$ for each $f \in \ell^{1}(I, v)$ where the sum converges in the norm $\|\cdot\|_{v}$. It is easy to see that $\left(\ell^{1}(I, v),\|\cdot\|_{v}\right)$ is a Banach space. In line with the general convention, we will simply write $\ell^{1}(I, v)$ for this Banach space, and $\ell^{1}(I)$ whenever $v=1$.

When $I$ is a monoid, there is a canonical way of turning $\ell^{1}(I, v)$ into a unital Banach algebra. Slightly more generally, let $S$ be a semigroup. Let $\omega: S \rightarrow(0,+\infty)$ be a weight on $S$, that is, we require $\omega(s t) \leq \omega(s) \omega(t)$ to hold for all $s, t \in S$. In addition, when $S$ is a monoid with multiplicative identity $e \in S$ then we also require $\omega(e)=1$. We remark that any weight is equivalent to one satisfying this normalisation condition. We define the usual convolution product on $\ell^{1}(S, \omega)$ by

$$
(f * g)(r):=\sum_{s t=r} f(s) g(t) \quad\left(f, g \in \ell^{1}(S, \omega), r \in S\right),
$$

then $\left(\ell^{1}(S, \omega), *\right)$ is a Banach algebra (one uses the condition on the weight to show that the norm is submultiplicative.) When $S$ is a monoid, $\left(\ell^{1}(S, \omega), *\right)$ becomes a unital Banach algebra with unit $\delta_{e}$, clearly $\left\|\delta_{e}\right\|_{\omega}=1$ holds.

In what follows, we shall mostly be interested in weights $\omega$ with $\omega(s) \geq 1$ for all $s$. Notice then that $\ell^{1}(S, \omega)$ becomes a (in general, not closed) subalgebra of $\ell^{1}(S)$.

Proposition 3.6 Let $S$ be a monoid with unit $e \in S$ and let $\omega: S \rightarrow[1,+\infty)$ be a weight on $S$. Let $p \in\left(\ell^{1}(S, \omega), *\right)$ be a non-zero idempotent such that $p \neq \delta_{e}$. Then

$$
\|p\|_{\omega} \geq \frac{1}{2} \inf \{\omega(s): s \in S, s \neq e\} .
$$

Proof Since $\left(\ell^{1}(S, \omega), *\right)$ is a subalgebra of $\left(\ell^{1}(S), *\right)$, we have that $p \in\left(\ell^{1}(S), *\right)$. Assume first that $p(e) \neq 0$. We claim that then $\left\|\delta_{e}-(p(e))^{-1} p\right\| \geq 1$. Indeed otherwise $\left\|\delta_{e}-(p(e))^{-1} p\right\|<1$ and thus $(p(e))^{-1} p$, and so $p$, are invertible in $\left(\ell^{1}(S), *\right)$, which is impossible as $p$ is an idempotent different from $\delta_{e}$. Consequently

$$
|p(e)| \leq|p(e)|\left\|\delta_{e}-\frac{1}{p(e)} p\right\|=\sum_{s \in S \backslash\{e\}}|p(s)| .
$$


If $p(e)=0$ then the above inequality obviously holds. As $p$ is an idempotent, we have $1 \leq\|p\|$, and this yields

$$
1 \leq\|p\|=|p(e)|+\sum_{s \in S \backslash\{e\}}|p(s)| \leq 2\left(\sum_{s \in S \backslash\{e\}}|p(s)|\right) .
$$

From this we conclude

$$
\begin{aligned}
\|p\|_{\omega} & =\sum_{s \in S}|p(s)| \omega(s) \geq \sum_{s \in S \backslash\{e\}}|p(s)| \omega(s) \\
& \geq \inf _{s \in S \backslash\{e\}} \omega(s) \sum_{s \in S \backslash\{e\}}|p(s)| \geq \frac{1}{2} \inf _{s \in S \backslash\{e\}} \omega(s) .
\end{aligned}
$$

In what follows $B C$ denotes the bicyclic monoid, which is the free monoid generated by elements $p, q$ subject to the single relation that $p q=e$ :

$$
B C=\langle p, q: p q=e\rangle .
$$

Fix $n \in \mathbb{N}$. Let $\omega_{n}: B C \rightarrow[1,+\infty)$ be the weight on $B C$ defined as $\omega_{n}(e)=1$ and $\omega_{n}(s)=n$ for $s \in B C \backslash\{e\}$.

Theorem 3.7 Let $\mathcal{A}_{n}:=\left(\ell^{1}\left(B C, \omega_{n}\right), *\right)$ for every $n \in \mathbb{N}$. Then $\left(\mathcal{A}_{n}\right)$ is a sequence of Dedekind-infinite Banach algebras such that $\operatorname{Asy}\left(\mathcal{A}_{n}\right)$ is Dedekind-finite.

Proof For any $n \in \mathbb{N}$, work in $\mathcal{A}_{n}$, and consider $h:=\delta_{q} * \delta_{p}$. Then $h$ is an idempotent with $h \sim \delta_{e}$ and $h \neq \delta_{e}$. Indeed, $\delta_{p} * \delta_{q}=\delta_{p q}=\delta_{e}$ and $\delta_{q} * \delta_{p}=\delta_{q p} \neq \delta_{e}$. This in particular shows that $\mathcal{A}_{n}$ is Dedekind-infinite.

Now let $h \in \mathcal{A}_{n}$ be an arbitrary idempotent such that $h \sim \delta_{e}$ and $h \neq \delta_{e}$. We observe that Proposition 3.6 yields $\|h\|_{\omega_{n}} \geq n / 2$, and consequently $C_{\mathrm{DI}}^{\prime}\left(\mathcal{A}_{n}\right) \geq n / 2$. In view of Corollary 3.5 the Banach algebra $\operatorname{Asy}\left(\mathcal{A}_{n}\right)$ is Dedekind-finite.

In particular, this shows that the converse of Theorem 3.1 does not hold in general. As we can vary finitely many of the $\mathcal{A}_{n}$ without changing $\operatorname{Asy}\left(\mathcal{A}_{n}\right)$, by using the contrapositive, we can alternatively state Theorem 3.1 as: if $\operatorname{Asy}\left(\mathcal{A}_{n}\right)$ is Dedekind-infinite, then infinitely many of the $\mathcal{A}_{n}$ are Dedekind-infinite. If we add in control of $C_{\mathrm{DI}}$ then we obtain a converse.

Proposition 3.8 Let $\left(\mathcal{A}_{n}\right)$ be a sequence of unital Banach algebras such that there exists $K>0$ and an increasing sequence $\left(n_{k}\right)$ in $\mathbb{N}$ with $C_{D I}\left(\mathcal{A}_{n_{k}}\right) \leq K$ for each $k \in \mathbb{N}$. Then $\operatorname{Asy}\left(\mathcal{A}_{n}\right)$ is Dedekind-infinite.

Proof By assumption, for each $k \in \mathbb{N}$ we can find $a_{n_{k}}, b_{n_{k}} \in \mathcal{A}_{n_{k}}$ with $\left\|a_{n_{k}}\right\|\left\|b_{n_{k}}\right\| \leq$ $K+1$, say, and $a_{n_{k}} b_{n_{k}}=1_{n_{k}}$ while $b_{n_{k}} a_{n_{k}} \neq 1_{n_{k}}$. We can rescale and suppose that $\left\|a_{n_{k}}\right\|=\left\|b_{n_{k}}\right\|$. For $n \in \mathbb{N}$ not in the sequence, define $a_{n}=b_{n}=1_{n}$. Then $A=$ $\left(a_{n}\right) \in \ell^{\infty}\left(\mathcal{A}_{n}\right)$, and similarly for $B=\left(b_{n}\right)$, and clearly $\pi(A) \pi(B)=1$. By Remark 3.3, 
we have that $\left\|b_{n_{k}} a_{n_{k}}-1_{n_{k}}\right\| \geq 1$ for each $k$, and so $\pi(B) \pi(A) \neq 1$. Thus $\operatorname{Asy}\left(\mathcal{A}_{n}\right)$ is Dedekind-infinite.

Furthermore, under certain conditions, we do obtain a direct converse to Theorem 3.1.

Corollary 3.9 Let $\left(\mathcal{A}_{n}\right)$ be a sequence of unital Banach algebras such that $\operatorname{Asy}\left(\mathcal{A}_{n}\right)$ is Dedekind-finite. Moreover, suppose that one of the following two conditions hold:

(1) $\mathcal{A}_{n}=\mathcal{A}_{m}$ for every $n, m \in \mathbb{N}$;

(2) $\mathcal{A}_{n}$ is a $C^{*}$-algebra for each $n \in \mathbb{N}$.

Then there is $N \in \mathbb{N}$ such that $\mathcal{A}_{n}$ is Dedekind-finite for $n \geq N$.

Proof If $\mathcal{A}_{n}=\mathcal{A}$ for each $n$, then if $\mathcal{A}$ is Dedekind-infinite, then $C_{\mathrm{DI}}(\mathcal{A})<\infty$ and so Proposition 3.8 shows that $\operatorname{Asy}\left(\mathcal{A}_{n}\right)$ is Dedekind-infinite, a contradiction.

Consider now a $C^{*}$-algebra $\mathcal{B}$. By Proposition 2.5, we know that $\mathcal{B}$ is Dedekindfinite if and only if it is Dedekind-finite in the $C^{*}$-algebraic sense, that is, if $u$ is a partial isometry with $u^{*} u=1$ then $u u^{*}=1$. It follows that $C_{\mathrm{DI}}(\mathcal{B})=1$ or $+\infty$. Thus, if each $\mathcal{A}_{n}$ is a $C^{*}$-algebra, and $\operatorname{Asy}\left(\mathcal{A}_{n}\right)$ is Dedekind-finite, then Proposition 3.8 shows that $C_{D I}\left(\mathcal{A}_{n}\right)=+\infty$ for all but finitely many $n$. That is, eventually $\mathcal{A}_{n}$ is Dedekind-finite, as claimed.

In the proof of Proposition 3.8, it seemed important to work with $C_{\mathrm{DI}}$ and not $C_{\mathrm{DI}}^{\prime}$. In fact this is necessary, as we now show. For the following, we need some simple combinatorics of the monoid $B C$. Any element of $B C$ can be written as a reduced word in the generators $p, q$, which is necessarily of the form $q^{\alpha} p^{\beta}$ with $\alpha, \beta \in \mathbb{N}_{0}$. The multiplication law is that

$$
\left(q^{\alpha} p^{\beta}\right)\left(q^{\gamma} p^{\delta}\right)= \begin{cases}q^{\alpha} p^{\delta+\beta-\gamma} & \text { if } \beta \geq \gamma \\ q^{\alpha-\beta+\gamma} p^{\delta} & \text { if } \beta \leq \gamma\end{cases}
$$

From this, it is easy to see that the set of idempotents in $B C$ is $B C_{I}=\left\{q^{\alpha} p^{\alpha}: \alpha \geq 0\right\}$. The following are also easy to see:

(1) $\left(q^{\alpha} p^{\alpha}\right)\left(q^{\beta} p^{\beta}\right)=q^{\gamma} p^{\gamma}$ where $\gamma=\max (\alpha, \beta)$;

(2) if $s \in B C_{I}, t \notin B C_{I}$ then $s t, t s \notin B C_{I}$.

By point (1) we see that $B C_{I}$ is a sub-semigroup of $B C$, and so we may consider $\ell^{1}\left(B C_{I}\right)$, which can be identified with a closed subalgebra of $\ell^{1}(B C)$.

Lemma 3.10 Let $S:=\left\{s_{n}\right\}$ be a countable semigroup consisting of idempotents such that $s_{n} s_{m}=s_{\max (n, m)}$ for every $n, m \in \mathbb{N}$. Then $f \in \ell^{1}(S)$ is an idempotent if and only if $f\left(s_{n}\right) \in\{-1,0,1\}$ for all $n$, there is an $n_{0} \geq 0$ so that $f\left(s_{n}\right)=0$ for $n \geq n_{0}$, and for all $m \geq 0$ we have that $\sum_{n=0}^{m} f\left(s_{n}\right) \in\{0,1\}$. 
Proof Firstly, let $f$ be an idempotent. To ease notation, let $e_{n}:=\delta_{s_{n}}$ and $f(n):=$ $f\left(s_{n}\right)$, so that $f$ has the expansion $f=\sum_{n \geq 0} f(n) e_{n}$. Then

$$
f^{2}=\sum_{n, m \geq 0} f(n) f(m) e_{\max (n, m)}=f \quad \Longrightarrow \quad \sum_{\max (n, m)=t} f(n) f(m)=f(t) \quad(t \geq 0) .
$$

Hence $f(0)^{2}=f(0)$ and so $f(0) \in\{0,1\}$. We now use strong induction: suppose that $f(n) \in\{-1,0,1\}$ for each $n \leq N$ and that $\sum_{n=0}^{m} f(n) \in\{0,1\}$ for each $m \leq N$. Then

$$
f(N+1)=2 f(N+1) \sum_{n=0}^{N} f(n)+f(N+1)^{2} .
$$

Either $\sum_{n=0}^{N} f(n)=0$, in which case $f(N+1) \in\{0,1\}$, and so $\sum_{n=0}^{N+1} f(n) \in\{0,1\}$; or alternatively $\sum_{n=0}^{N} f(n)=1$ in which case $0=f(N+1)+f(N+1)^{2}$ so $f(N+1) \in\{0,-1\}$ and so $\sum_{n=0}^{N+1} f(n) \in\{0,1\}$. Finally, as $\sum_{n=0}^{\infty} f(n)$ must converge, we must have that $f(n)=0$ eventually.

We now consider the converse. Given such an $f$, we have that

$$
\sum_{\max (n, m)=t} f(n) f(m)=2 f(t) \sum_{n<t} f(n)+f(t)^{2} \quad(t \geq 1) .
$$

Either $\sum_{n<t} f(n)=0$, in which case, as $\sum_{n \leq t} f(n) \in\{0,1\}$ we must have that $f(t) \in$ $\{0,1\}$, and so $f(t)^{2}=f(t)$ as required; or $\sum_{n<t} f(n)=1$, in which case, as $\sum_{n \leq t} f(n) \in$ $\{0,1\}$ we must have that $f(t) \in\{-1,0\}$, and so $2 f(t) \sum_{n<t} f(n)+f(t)^{2}=f(t)(2+$ $f(t))=f(t)$ for either $f(t)=0$ or $f(t)=-1$, as required.

Proposition 3.11 Let $\omega$ be a weight on $B C$ such that $\omega \geq 1$ and $\omega(s) \geq N$ for each $s \notin B C_{I}$. Then $C_{D I}\left(\ell^{1}(B C, \omega)\right) \geq(N / 86)^{1 / 3}$.

Proof Let $\mathcal{A}=\ell^{1}(B C, \omega)$, and suppose that $K>C_{\mathrm{DI}}(\mathcal{A})$, so that $K>1$, and we can find $f, g \in \mathcal{A}$ such that $f * g=\delta_{e}$ and $g * f \neq \delta_{e}$, and with $\|f\|_{\omega}\|g\|_{\omega} \leq K$. As $\omega \geq 1$ we can regard $\mathcal{A}$ as a (possibly not closed) subalgebra of $\ell^{1}(B C)$.

Let $h:=g * f$, then $h$ is an idempotent. Let $k \in \ell^{1}\left(B C_{I}\right)$ be the restriction of $h$ onto $B C_{I}$. Working in $\ell^{1}(B C)$, we notice that

$$
\|h\|_{\omega}=\sum_{s \in B C_{I}}|h(s)| \omega(s)+\sum_{s \notin B C_{I}}|h(s)| \omega(s) \geq N \sum_{s \notin B C_{I}}|h(s)|=N\|h-k\|,
$$

by our assumption on the weight. We now observe that, because $h^{2}=h$,

$$
\begin{aligned}
\left\|k^{2}-k\right\| & =\|k(k-h)+(k-h) h+h-k\| \\
& \leq(\|k\|+\|h\|+1)\|k-h\| \leq \frac{\|h\|_{\omega}}{N}\left(2\|h\|_{\omega}+1\right) .
\end{aligned}
$$

Let this quantity be $v$, and suppose that $v<1 / 4$. Working in $\ell^{1}\left(B C_{I}\right)$, by Proposition 2.6 there is an idempotent $k^{\prime} \in \ell^{1}\left(B C_{I}\right)$ with

$$
\left\|k-k^{\prime}\right\| \leq(\|k\|+1 / 2)\left((1-4 v)^{-1 / 2}-1\right) .
$$


Let this quantity be $v^{\prime}$. Working in $\ell^{1}(B C)$, we have that

$$
\left\|h-k^{\prime}\right\| \leq\|h-k\|+\left\|k-k^{\prime}\right\| \leq v^{\prime}+\frac{\|h\|_{\omega}}{N} .
$$

Let this quantity be $\epsilon$.

Assume that $\epsilon<1$. By Remark 3.3, as $h \neq \delta_{e}$, we have that $\left\|\delta_{e}-h\right\| \geq 1$, and so $k^{\prime} \neq \delta_{e}$.

Let $e_{n}=\delta_{q^{n} p^{n}}$ for all $n \in \mathbb{N}_{0}$ and write

$$
k^{\prime}=\sum_{n \geq 0} k^{\prime}(n) e_{n}
$$

As $k^{\prime} \in \ell^{1}\left(B C_{I}\right)$ is an idempotent, we hence conclude, using Lemma 3.10, that there is some $t \geq 1$ with $k^{\prime}(t)= \pm 1$ and so certainly $|h(t) \mp 1|<\epsilon$.

Write

$$
f=\sum_{n \geq 0} f(n) e_{n}+f^{\prime}=f^{\prime \prime}+f^{\prime}
$$

say, where $f^{\prime}$ is supported off $B C_{I}$, and similarly for $g$. Then

$$
f * g=\sum_{n, m \geq 0} f(n) g(m) e_{\max (n, m)}+f^{\prime} * g^{\prime \prime}+f^{\prime \prime} * g^{\prime}+f^{\prime} * g^{\prime} .
$$

Notice that by the points above Lemma 3.10, we have that $f^{\prime} * g^{\prime \prime}+f^{\prime \prime} * g^{\prime}$ is supported off $B C_{I}$. Write $\left(f^{\prime} g^{\prime}\right)_{n}$ for the coefficient of $e_{n}$ in the expansion of $f^{\prime} * g^{\prime}$, and similarly for $g^{\prime} * f^{\prime}$. That $f * g=\delta_{e}$ means that

$$
\sum_{\max (n, m)=t} f(n) g(m)+\left(f^{\prime} g^{\prime}\right)_{t}=0 \quad(t \geq 1) .
$$

Also, $|h(t) \mp 1|<\epsilon$ and that $g^{\prime} * f^{\prime \prime}+g^{\prime \prime} * f^{\prime}$ is supported off $B C_{I}$ means that

$$
\left|\sum_{\max (n, m)=t} f(n) g(m)+\left(g^{\prime} f^{\prime}\right)_{t} \mp 1\right|<\epsilon \quad(t \geq 1) .
$$

Using also (3.16) we see that $\left|-\left(f^{\prime} g^{\prime}\right)_{t}+\left(g^{\prime} f^{\prime}\right)_{t} \mp 1\right|<\epsilon$. Thus $\left|\left(g^{\prime} f^{\prime}\right)_{t}-\left(f^{\prime} g^{\prime}\right)_{t}\right|>1-\epsilon$, and so $\left\|f^{\prime}\right\|\left\|g^{\prime}\right\| \geq(1-\epsilon) / 2$. It follows that

$$
K \geq\|f\|_{\omega}\|g\|_{\omega} \geq N^{2}\left\|f^{\prime}\right\|\left\|g^{\prime}\right\| \geq \frac{N^{2}}{2}(1-\epsilon) .
$$

Choose $\epsilon_{0} \in(0,1)$, and suppose that $43 K^{3} \leq N \epsilon_{0}$, so certainly $K^{2}<N / 15$. As $\|h\|_{\omega} \leq K$, we see that $v \leq K(2 K+1) / N \leq 3 K^{2} / N<1 / 5$, and so $(1-4 v)^{-1 / 2}-1 \leq 7 v$, so that

$$
v^{\prime} \leq(K+1 / 2)\left((1-4 v)^{-1 / 2}-1\right) \leq 14 K v \leq \frac{42 K^{3}}{N} .
$$

Finally, $\epsilon=v^{\prime}+\|h\|_{w} / N \leq\left(42 K^{3}+K\right) / N \leq 43 K^{3} / N \leq \epsilon_{0}$. We conclude that if $43 K^{3} \leq N \epsilon_{0}$ then $N^{2}\left(1-\epsilon_{0}\right) / 2 \leq K$.

Now, $K>C_{\mathrm{DI}}(\mathcal{A})$ is arbitrary, so take $\epsilon_{0}=1 / 2$, for example, to conclude that if $C_{\text {DI }}(\mathcal{A})<(N / 86)^{1 / 3}$, then we can choose a suitable $K \leq\left(N \epsilon_{0} / 43\right)^{1 / 3}$, and so conclude 
that

$$
\frac{N^{2}}{2}\left(1-\epsilon_{0}\right)=\frac{N^{2}}{4} \leq K \leq(N / 86)^{1 / 3} \quad \Longrightarrow \quad N^{5} \leq \frac{64}{86},
$$

which is a contradiction, as required.

Proposition 3.12 For any $n \in \mathbb{N}$ we can find a unital Banach algebra $\mathcal{A}_{n}$ with $C_{D I}^{\prime}\left(\mathcal{A}_{n}\right)=$ 1 yet $C_{D I}\left(\mathcal{A}_{n}\right) \geq n$.

Proof Given $n$, pick $N$ with $N \geq 86 n^{3}$. Define $\omega_{n}$ on $B C$ by $\omega_{n}(e)=1, \omega_{n}(q p)=1$, $\omega_{n}(s)=N$ otherwise. Let $X=\{e, q p\} \subseteq B C$. We now prove that $\omega_{n}$ is a weight, for which we need to show that $\omega_{n}(s t) \leq \omega_{n}(s) \omega_{n}(t)$ for all $s, t \in B C$. This can only fail if we can find $s, t$ such that $\omega_{n}(s t)=N$ yet $\omega_{n}(s)=\omega_{n}(t)=1$, which is impossible as $X$ is a sub-semigroup of $B C$.

Now consider $\mathcal{A}_{n}:=\ell^{1}\left(B C, \omega_{n}\right)$, and let $h=\delta_{q p} \in \mathcal{A}_{n}$. Then $\|h\|_{\omega_{n}}=1$ and as before, $h \sim \delta_{e}$, so $C_{\mathrm{DI}}^{\prime}\left(\mathcal{A}_{n}\right) \leq 1$, hence $C_{\mathrm{DI}}^{\prime}\left(\mathcal{A}_{n}\right)=1$. However, by Proposition 3.11, $C_{\mathrm{DI}}\left(\mathcal{A}_{n}\right) \geq(N / 86)^{1 / 3} \geq n$, as required.

Using this proposition, we thus obtain a sequence $\left(\mathcal{A}_{n}\right)$ of Dedekind-infinite Banach algebras, with $C_{\mathrm{DI}}^{\prime}\left(\mathcal{A}_{n}\right)=1$ for each $n$, but with $C_{\mathrm{DI}}\left(\mathcal{A}_{n}\right) \rightarrow \infty$. By Proposition 3.4 we find that $\operatorname{Asy}\left(\mathcal{A}_{n}\right)$ is Dedekind-finite. We conclude that in Proposition 3.8 we cannot replace $C_{\mathrm{DI}}$ with $C_{\mathrm{DI}}^{\prime}$.

Remark 3.13 Notice also that in this way, we obtain a sequence $\left(\mathcal{A}_{n}\right)$ of Banach algebras, and idempotents $p_{n} \in \mathcal{A}_{n}$ such that each $p_{n}$ is equivalent to $1_{n}$, but the (equivalence class of the) sequence $P=\left(p_{n}\right)$ is not equivalent to 1 in $\operatorname{Asy}\left(\mathcal{A}_{n}\right)$.

Remark 3.14 The weights we have so far constructed have the property that $\ell^{1}\left(B C, \omega_{n}\right)$ is isomorphic (just not isometric) with $\ell^{1}(B C)$, for the formal identity map. However, we can easily construct examples which do not have this property.

Viewing $B C$ as the set of reduced words of the form $s=q^{\alpha} p^{\beta}$ we define the word length as $\ell(s)=\alpha+\beta$. It is easy to see that $\ell: B C \rightarrow \mathbb{N}_{0}$ is sub-additive: $\ell(s t) \leq$ $\ell(s)+\ell(t)$. As such, $\omega_{x}(s)=\exp (x \ell(s))$ defines a weight for every $x \geq 0$. Furthermore, $\ell^{1}\left(B C, \omega_{x}\right)$ is not (naturally) isomorphic to $\ell^{1}(B C)$ for any $x>0$.

We now adapt the construction in the proof of Proposition 3.12. Define, for $x \geq 0$,

$$
\omega_{x}(s)= \begin{cases}1 & \text { if } s=e \text { or } s=q p, \\ \exp (x \ell(s)) & \text { otherwise. }\end{cases}
$$

This is a weight by the same argument as in the proof of Proposition 3.12. As before, with $\mathcal{A}_{x}=\ell^{1}\left(B C, \omega_{x}\right)$, we have $C_{\mathrm{DI}}^{\prime}\left(\mathcal{A}_{x}\right)=1$ while $C_{\mathrm{DI}}\left(\mathcal{A}_{x}\right) \geq\left(e^{x} / 86\right)^{1 / 3}$.

\subsection{Renormings}

In Section 2 we mentioned that any unital Banach algebra can be renormed so as to make the norm of the unit be 1. Let us explore this further. Firstly, we could take a more "permissive" definition of a unital Banach algebra: a complete normed algebra, with a 
contractive product, and an element $e \in \mathcal{A}$ with $e a=a e=a$ for each $a \in \mathcal{A}$. That is, we do not assume that $\|e\|=1$. Notice that if $\mathcal{A}$ is a Banach algebra with a unit of norm one, then for $m \geq 1$ we can define $\|a\|_{m}=m\|a\|$ for $a \in \mathcal{A}$. Then $\|\cdot\|_{m}$ is an equivalent norm on $\mathcal{A}$, and as $\|a b\|_{m}=m\|a b\| \leq m\|a\|\|b\| \leq m^{2}\|a\|\|b\|=\|a\|_{m}\|b\|_{m}$, we have a contractive product, but of course now $\|e\|_{m}=m$.

Proposition 3.15 With this definition of a unital Banach algebra, there exists a sequence $\left(\mathcal{A}_{n}\right)$ of unital Banach algebras such that $\operatorname{Asy}\left(\mathcal{A}_{n}\right)$ is not unital.

Proof Fix a unital Banach algebra $\mathcal{A}$ with a norm one unit $e$. For each $n \in \mathbb{N}$ let $\mathcal{A}_{n}=\left(\mathcal{A},\|\cdot\|_{n}\right)$ with $\|\cdot\|_{n}$ defined as above. Towards a contradiction, suppose that $f \in \operatorname{Asy}\left(\mathcal{A}_{n}\right)$ is a unit, say $f=\pi(F)$ with $F=\left(f_{n}\right) \in \ell^{\infty}\left(\mathcal{A}_{n}\right)$, so that

$$
0=\limsup _{n \rightarrow \infty}\left\|a_{n} f_{n}-a_{n}\right\|_{n}=\limsup _{n \rightarrow \infty} n\left\|a_{n} f_{n}-a_{n}\right\|
$$

for each $A=\left(a_{n}\right) \in \ell^{\infty}\left(\mathcal{A}_{n}\right)$. Notice that $\left(a_{n}\right) \in \ell^{\infty}\left(\mathcal{A}_{n}\right)$ exactly when there is $K>0$ with $\left\|a_{n}\right\| \leq K n^{-1}$ for each $n$. Let $b_{n}:=e / n$ for all $n \in \mathbb{N}$. Then $\left\|b_{n}\right\|=1 / n$ and $\left\|b_{n}\right\|_{n}=1$, hence $B:=\left(b_{n}\right) \in \ell^{\infty}\left(\mathcal{A}_{n}\right)$.

We hence see that

$$
0=\limsup _{n \rightarrow \infty} n\left\|b_{n} f_{n}-b_{n}\right\|=\limsup _{n \rightarrow \infty}\left\|f_{n}-e\right\| .
$$

However, as $\left\|f_{n}\right\| \leq K n^{-1}$ for some fixed $K$, we see that $\left\|e-f_{n}\right\| \geq\|e\|-\left\|f_{n}\right\| \geq$ $1-K / n$ which is a contradiction.

We thus see that it pays to be careful about "implicit renormings", when considering the sort of questions we are asking. We are also now lead to wonder how the constants $C_{\mathrm{DI}}(\mathcal{A})$ and $C_{\mathrm{DI}}^{\prime}(\mathcal{A})$ behave under renormings. The following is a result in this direction; we remark that we revert to our standing assumption that unital Banach algebras have contractive multiplications and units of norm one.

Proposition 3.16 Let $\mathcal{A}$ be a Dedekind-infinite Banach algebra. There is an equivalent norm $\|\cdot\|_{0}$ on $\mathcal{A}$ such that $\left(\mathcal{A},\|\cdot\|_{0}\right)$ is a unital Banach algebra, and $C_{D I}^{\prime}\left(\mathcal{A},\|\cdot\|_{0}\right)=1$.

Proof Let $p \in \mathcal{A}$ be an idempotent with $p \sim 1$ and $p \neq 1$. The set $S:=\{1, p\}$ is clearly a bounded sub-semigroup of $\mathcal{A}$. Hence by [3, Proposition 2.1.9] there is a submultiplicative norm $\|\cdot\|_{0}$ equivalent to $\|\cdot\|$ on $\mathcal{A}$ such that $\left(\mathcal{A},\|\cdot\|_{0}\right)$ is Banach algebra and $\|s\|_{0} \leq 1$ for every $s \in S$. In particular $\|1\|_{0} \leq 1$ and $\|p\|_{0} \leq 1$, hence $\|1\|_{0}=1$ and $\|p\|_{0}=1$. Consequently $C_{D I}^{\prime}\left(\mathcal{A},\|\cdot\|_{0}\right)=1$.

For the benefit of the reader, we remark that [3, Proposition 2.1.9] is proved as follows. Firstly we define $v(a)=\sup \{\|a\|,\|s a\|: s \in S\}$ and then notice that $v$ is an equivalent norm on $\mathcal{A}$ with bounded product, such that $S$ is $v$-bounded, and such that $v(s a) \leq v(a)$ for $a \in \mathcal{A}, s \in S$. Then let $E$ be the unconditional unitisation of $(\mathcal{A}, v)$, and finally let $\|\cdot\|_{0}$ be the norm induced on $\mathcal{A}$ by the left-regular representation of $\mathcal{A}$ on $E$. 
Remark 3.17 For each $K \geq 1$, we have examples of $\mathcal{A}$ where $C_{\mathrm{DI}}^{\prime}(\mathcal{A}) \geq K$, see the proof of Theorem 3.7 for example. Thus, in the previous proposition, while the original norm $\|\cdot\|$ and the new norm $\|\cdot\|_{0}$ are equivalent, there is not some absolute constant $C>0$ (independent of $\mathcal{A}$ ) with $C^{-1}\|\cdot\| \leq\|\cdot\|_{0} \leq C\|\cdot\|$.

Notice that in the above proof, we actually showed something more: given any unital Banach algebra $\mathcal{A}$ with an idempotent $p \in \mathcal{A}$ such that $p \sim 1$ and $p \neq 1$, we can find an equivalent norm $\|\cdot\|_{0}$ on $\mathcal{A}$ with $\|p\|_{0}=1$, hence $p$ witnesses that $C_{D I}^{\prime}\left(\mathcal{A},\|\cdot\|_{0}\right)=1$. The following shows that a similar statement does not hold for $C_{\mathrm{DI}}$.

Proposition 3.18 For each $K>0$ there is a Banach algebra $\mathcal{A}$ and $a, b \in \mathcal{A}$ with $a b=$ $1, b a \neq 1$, and such that, if $\|\cdot\|_{0}$ is any equivalent norm on $\mathcal{A}$, then $\|a\|_{0}\|b\|_{0} \geq K$.

Proof We use the same weight $\omega_{x}$ as in Remark 3.14, and set $\mathcal{A}_{x}=\ell^{1}\left(B C, \omega_{x}\right)$. Let $\|\cdot\|_{0}$ be an equivalent norm on $\mathcal{A}_{x}$, so there is $m>0$ with $m^{-1}\|f\|_{\omega_{x}} \leq\|f\|_{0} \leq$ $m\|f\|_{\omega_{x}}$ for all $f \in \mathcal{A}_{x}$. Let $s=q^{\alpha} p^{\beta} \in B C$, with say $\alpha>\beta$. Then

$$
s^{2}=q^{2 \alpha-\beta} p^{\beta}, \quad s^{3}=q^{3 \alpha-2 \beta} p^{\beta}, \quad \cdots, \quad s^{n}=q^{n \alpha-(n-1) \beta} p^{\beta} .
$$

It follows that

$$
\begin{aligned}
& m^{-1} \exp (x(n \alpha-(n-2) \beta))=m^{-1}\left\|\delta_{s^{n}}\right\|_{\omega_{x}} \leq\left\|\delta_{s^{n}}\right\|_{0} \leq\left\|\delta_{s}\right\|_{0}^{n} \\
\Longrightarrow & \frac{-\log (m)}{n}+x \frac{n \alpha-(n-2) \beta}{n} \leq \log \left\|\delta_{s}\right\|_{0} \\
\Longrightarrow \quad & \log \left\|\delta_{s}\right\|_{0} \geq \liminf _{n \rightarrow \infty}\left(\frac{-\log (m)}{n}+x \frac{n \alpha-(n-2) \beta}{n}\right)=x(\alpha-\beta) .
\end{aligned}
$$

Thus $\left\|\delta_{s}\right\|_{0} \geq e^{x(\alpha-\beta)}$. The same argument applies in the case when $\alpha<\beta$.

As in the proof of Proposition 3.11, set $a=\delta_{p}$ and $b=\delta_{q}$, so that $a b=1$ and $b a \neq 1$. We have just shown that $\|a\|_{0} \geq e^{x}$ and $\|b\|_{0} \geq e^{x}$, which completes the proof.

We have been unable to decide if it is possible to renorm $\mathcal{A}_{x}$ so as to get $C_{\mathrm{DI}}\left(\mathcal{A}_{x}, \| \cdot\right.$ $\left.\|_{0}\right)=1$ (or just be smaller than some absolute constant). The difficulty is that, given the previous proposition, we need consider the possibility of some other elements $c, d \in \mathcal{A}_{x}$ with $c d=1$ and $d c \neq 1$, while also considering an arbitrary renorming.

\subsection{For ultraproducts}

All of these results hold for ultraproducts with suitable modifications. Let us start by indicating how to give a "bare-hands" proof.

Theorem 3.19 Let $\left(\mathcal{A}_{n}\right)$ be a sequence of Banach algebras, and let $\mathcal{U}$ be an ultrafilter such that $\left\{n \in \mathbb{N}: \mathcal{A}_{n}\right.$ is Dedekind-finite $\} \in \mathcal{U}$. Then $\left(\mathcal{A}_{n}\right) \mathcal{U}$ is Dedekind-finite.

Proof We simply adapt the proof of Theorem 3.1. Firstly, we find that there is $U \in \mathcal{U}$ with $n \in U \Longrightarrow v_{n}=\left\|x_{n}-x_{n}^{2}\right\|<1 / 8$. This allows us to find $P=\left(p_{n}\right)$ so that $\pi(P)=p \in\left(\mathcal{A}_{n}\right) \mathcal{U}$. Then we find $U^{\prime} \subseteq U$ with $U^{\prime} \in \mathcal{U}$ and $u_{n}^{-1}$ existing for $n \in U^{\prime}$. 
But moving to a smaller subset $U^{\prime \prime}$, we can assume that $\mathcal{A}_{n}$ is Dedekind-finite for each $n \in U^{\prime \prime}$. We then move finally to a yet smaller subset to finish the proof.

Proposition 3.4 and Corollary 3.5 also hold for ultraproducts. Indeed, given an ultrafilter $\mathcal{U}$, we only need the weaker condition that for each $N \in \mathbb{N}$ we have that $\{n \in \mathbb{N}$ : $\left.C_{\mathrm{DI}}\left(\mathcal{A}_{n}\right) \geq N\right\} \in \mathcal{U}$ (or for $\left.C_{\mathrm{DI}}^{\prime}\right)$. Hence also, with $\mathcal{A}_{n}:=\left(\ell^{1}\left(B C, \omega_{n}\right), *\right)$ for every $n$, we have that $\left(\mathcal{A}_{n}\right) \mathcal{U}$ is Dedekind-finite while each $\mathcal{A}_{n}$ is Dedekind-infinite. The analogue of Proposition 3.8 is the following.

Proposition 3.20 Let $\left(\mathcal{A}_{n}\right)$ be a sequence of unital Banach algebras, let $\mathcal{U}$ be an ultrafilter, and suppose that there exists $K>0$ such that $\left\{n \in \mathbb{N}: C_{D I}\left(\mathcal{A}_{n}\right) \leq K\right\} \in \mathcal{U}$. Then $\left(\mathcal{A}_{n}\right)_{\mathcal{U}}$ is Dedekind-infinite.

Let us finish this section by indicating how we could have used Łośs Theorem instead. We need to encode the property of being Dedekind-finite (with suitable "norm control") into the language of Banach algebras. In fact, it seems easier to work with Dedekind-infinite. The idea of the following is extracted from the proofs of Theorem 3.1 and Proposition 3.4.

Lemma 3.21 For $n \in \mathbb{N}$, let $\varphi_{n}$ be the sentence

$$
\inf _{a \in B_{n}} \inf _{b \in B_{n}} \max (\|a b-1\|, 1-\|b a-1\|) .
$$

Let $\mathcal{A}$ be a unital Banach algebra and let $\epsilon \in\left(0,1 / 3 n^{2}\right)$. If $\varphi_{n}^{\mathcal{A}}<\epsilon$ then $C_{D I}(\mathcal{A}) \leq$ $n^{2} /(1-\epsilon)$. Moreover, $\varphi_{n}^{\mathcal{A}}=0$ if and only if $C_{D I}(\mathcal{A}) \leq n^{2}$.

Proof Let $n \in \mathbb{N}$ be fixed throughout the proof. Assume $\varphi_{n}^{\mathcal{A}}<\epsilon$. There are $a, b \in \mathcal{A}$ with $\|a\|,\|b\| \leq n,\|a b-1\|<\epsilon$ and $1-\|b a-1\|<\epsilon$, so that $\|b a-1\|>1-\epsilon$. Let $u:=a b$, then by the Carl Neumann series $u \in \operatorname{inv}(\mathcal{A})$ with $\left\|1-u^{-1}\right\| \leq \epsilon /(1-\epsilon)$ and $\left\|u^{-1}\right\| \leq 1 /(1-\epsilon)$. Let $a^{\prime}:=u^{-1} a$ and $b^{\prime}:=b$, then $a^{\prime} b^{\prime}=1$ and $b^{\prime} a^{\prime}=b u^{-1} a$. We claim that $b^{\prime} a^{\prime} \neq 1$. For assume towards a contradiction that $b^{\prime} a^{\prime}=1$, so

$$
1-\epsilon<\|b a-1\| \leq\left\|b a-b u^{-1} a\right\| \leq\|a\|\|b\|\left\|1-u^{-1}\right\| \leq n^{2} \frac{\epsilon}{1-\epsilon}
$$

This is impossible because $\epsilon \in\left(0,1 / 3 n^{2}\right)$, and so we conclude that $b^{\prime} a^{\prime} \neq 1$. As $\left\|a^{\prime}\right\|\left\|b^{\prime}\right\| \leq\|a\|\|b\|(1-\epsilon)^{-1}$, we have that $C_{\mathrm{DI}}(\mathcal{A}) \leq n^{2} /(1-\epsilon)$.

For the second part of the statement suppose first $\varphi_{n}^{\mathcal{A}}=0$. Clearly $\varphi_{n}^{\mathcal{A}}<\epsilon$, hence $C_{\mathrm{DI}}(\mathcal{A}) \leq n^{2} /(1-\epsilon)$ for each $\epsilon \in\left(0,1 / 3 n^{2}\right)$ by the first part of the statement. Thus $C_{\mathrm{DI}}(\mathcal{A}) \leq n^{2}$.

Conversely, suppose $C_{\mathrm{DI}}(\mathcal{A}) \leq n^{2}$. Let us fix $\epsilon \in(0,1)$. We can find $a, b \in \mathcal{A}$ with $a b=1, b a \neq 1$ and $\|a\|\|b\| \leq(n+\epsilon)^{2}$. By rescaling, we may suppose that $\|a\|=$ $\|b\| \leq n+\epsilon$. As $b a \in \mathcal{A}$ is an idempotent not equal to 1 , by Remark 3.3 we know that $\|b a-1\| \geq 1$. Set $a^{\prime}:=n(n+\epsilon)^{-1} a$ and $b^{\prime}:=n(n+\epsilon)^{-1} b$, so that $\left\|a^{\prime}\right\|=\left\|b^{\prime}\right\| \leq n$. 
Let us observe that

$$
\begin{aligned}
&\left\|a^{\prime} b^{\prime}-1\right\|=\left|\frac{n^{2}}{(n+\epsilon)^{2}}-1\right|=\frac{\epsilon(2 n+\epsilon)}{(n+\epsilon)^{2}}<\epsilon(2 n+\epsilon), \\
&\left\|b^{\prime} a^{\prime}-b a\right\| \leq\left|\frac{n^{2}}{(n+\epsilon)^{2}}-1\right|\|b\|\|a\| \leq\left|\frac{n^{2}}{(n+\epsilon)^{2}}-1\right|(n+\epsilon)^{2}=\epsilon(2 n+\epsilon) .
\end{aligned}
$$

Consequently $\left\|b^{\prime} a^{\prime}-1\right\| \geq\|b a-1\|-\left\|b^{\prime} a^{\prime}-b a\right\|>1-\epsilon(2 n+\epsilon)$. We conclude that $\varphi_{n}^{\mathcal{A}}<\epsilon(2 n+\epsilon)$, and as $\epsilon \in(0,1)$ is arbitrary, we see that in fact $\varphi_{n}^{\mathcal{A}}=0$.

With the above result we can give alternative proofs to Theorems 3.19 and 3.20.

Proof We prove the contrapositive. Assume therefore $\left(\mathcal{A}_{i}\right)_{\mathcal{U}}$ is Dedekind-infinite, thus there is an $n \in \mathbb{N}$ such that $C_{\mathrm{DI}}\left(\left(\mathcal{A}_{i}\right) \mathcal{U}\right) \leq n^{2}$. By Lemma 3.21 this is equivalent to saying $\varphi_{n}^{\left(\mathcal{A}_{i}\right) \mathcal{U}}=0$, which in turn is equivalent to $\lim _{i \rightarrow \mathcal{U}} \varphi_{n}^{\mathcal{A}_{i}}=0$ by Łoś's Theorem. Thus for a fixed $\epsilon \in\left(0,1 / 3 n^{2}\right)$ we can find $U \in \mathcal{U}$ such that $\varphi_{n}^{\mathcal{A}_{i}}<\epsilon$ for each $i \in U$. Applying Lemma 3.21 again, we conclude that $C_{\mathrm{DI}}\left(\mathcal{A}_{i}\right) \leq n^{2} /(1-\epsilon)$ and hence $\mathcal{A}_{i}$ is Dedekind-infinite for each $i \in U$.

Proof We may (and do) assume that $K \in \mathbb{N}$ and $U:=\left\{i \in \mathbb{N}: C_{\mathrm{DI}}\left(\mathcal{A}_{i}\right) \leq K^{2}\right\} \in \mathcal{U}$. Then by Lemma 3.21 we see that $\varphi_{K}^{\mathcal{A}_{i}}=0$ for each $i \in U$. Hence by Łoś's Theorem we obtain $0=\lim _{i \rightarrow \mathcal{U}} \varphi_{K}^{\mathcal{A}_{i}}=\varphi_{K}^{\left(\mathcal{A}_{i}\right)_{\mathcal{U}}}$. In view of Lemma 3.21 again this is equivalent to $C_{\mathrm{DI}}\left(\left(\mathcal{A}_{i}\right)_{\mathcal{U}}\right) \leq K^{2}$, thus $\left(\mathcal{A}_{i}\right)_{\mathcal{U}}$ is Dedekind-infinite.

\section{Proper infiniteness}

Recall that a Banach algebra $\mathcal{A}$ is properly infinite when there exist idempotents $p \sim 1$ and $q \sim 1$ which are orthogonal, $p q=0=q p$.

\subsection{When the asymptotic sequence algebra is properly infinite}

The idea of the following proposition originates in [13].

Proposition 4.1 Let $\left(\mathcal{A}_{n}\right)$ be a sequence of unital Banach algebras, and let $p, q \in \operatorname{Asy}\left(\mathcal{A}_{n}\right)$ be idempotents with $[p, q]=0$. Then there exist idempotents $P, Q \in \ell^{\infty}\left(\mathcal{A}_{n}\right)$ with $p=\pi(P)$, $q=\pi(Q)$ and $[P, Q]=0$.

Proof Exactly as in the proof of Theorem 3.1, given an idempotent $p \in \operatorname{Asy}\left(\mathcal{A}_{n}\right)$, we can find an idempotent $P=\left(p_{n}\right) \in \ell^{\infty}\left(\mathcal{A}_{n}\right)$ with $\pi(P)=p$.

Now let $Y \in \ell^{\infty}\left(\mathcal{A}_{n}\right)$ be such that $q=\pi(Y)$. Let $Z:=(1-P) Y(1-P)+P Y P$, then from $[p, q]=0$ we obtain

$$
\begin{aligned}
\pi(Z) & =(1-p) q(1-p)+p q p \\
& =q-p q-q p+p q p+p q p=q-2 p q+2 p^{2} q=q .
\end{aligned}
$$

It is clear that $[P, Z]=0$, so if we write $Z=\left(z_{n}\right)$, then $\left[p_{n}, z_{n}\right]=0$ for every $n \in \mathbb{N}$. 
As $\pi(Z)=q$, we see that $q=q^{2}$ is equivalent to $\lim _{n \rightarrow \infty}\left\|z_{n}-z_{n}^{2}\right\|=0$. Let $\mu_{n}:=$ $\left\|z_{n}-z_{n}^{2}\right\|$ for every $n \in \mathbb{N}$. There is $M$ such that for every $n \geq M$ we have $\mu_{n}<1 / 8$. In view of Proposition 2.6, for every $n \geq M$ there is an idempotent $q_{n}^{\prime} \in \mathcal{A}_{n}$ with $\left\|z_{n}-q_{n}^{\prime}\right\| \leq f_{\left\|z_{n}\right\|}\left(\mu_{n}\right) \leq f_{\|Z\|}\left(\mu_{n}\right) \leq f_{\|Z\|}(1 / 8)$. Moreover, for every $n \in \mathbb{N}$ we have $\left[q_{n}^{\prime}, y\right]=0$ whenever $y \in \mathcal{A}_{n}$ is such that $\left[z_{n}, y\right]=0$. In particular, $\left[q_{n}^{\prime}, p_{n}\right]=0$ for all $n \geq M$.

By continuity of $f_{\|Z\|}$, it follows that $\lim _{n \geq N} f_{\|Z\|}\left(\mu_{n}\right)=0$; consequently $\lim _{n \geq N}\left\|z_{n}-q_{n}^{\prime}\right\|=0$. For every $n \in \mathbb{N}$ we define

$$
q_{n}:= \begin{cases}q_{n}^{\prime} & \text { if } n \geq M \\ 0 & \text { otherwise }\end{cases}
$$

Since $\left\|q_{n}^{\prime}\right\| \leq\left\|q_{n}^{\prime}-z_{n}\right\|+\left\|z_{n}\right\| \leq f_{\|Z\|}(1 / 8)+\|Z\|$ for all $n \geq M$, it follows that $Q:=$ $\left(q_{n}\right)$ is an idempotent in $\ell^{\infty}\left(\mathcal{A}_{n}\right)$. We observe that $q=\pi(Q)$ by $\lim _{n \geq M}\left\|z_{n}-q_{n}^{\prime}\right\|=0$. It is clear from the above that $[P, Q]=0$, thus concluding the proof.

Theorem 4.2 Let $\left(\mathcal{A}_{n}\right)$ be a sequence of unital Banach algebras such that $\operatorname{Asy}\left(\mathcal{A}_{n}\right)$ is properly infinite. Then there is an $N \in \mathbb{N}$ such that $\mathcal{A}_{n}$ is properly infinite for every $n \geq N$.

Proof Let $p, q \in \operatorname{Asy}\left(\mathcal{A}_{n}\right)$ be mutually orthogonal idempotents with $p, q \sim 1$. By Proposition 4.1 there exist idempotents $P, Q \in \ell^{\infty}\left(\mathcal{A}_{n}\right)$ with $p=\pi(P), q=\pi(Q)$ and $[P, Q]=0$. It follows from $p, q \sim 1$ that there exist $A=\left(a_{n}\right), B=\left(b_{n}\right), C=\left(c_{n}\right)$, $D=\left(d_{n}\right) \in \ell^{\infty}\left(\mathcal{A}_{n}\right)$ such that $1=\pi(A) \pi(B), p=\pi(B) \pi(A)$, and $1=\pi(C) \pi(D)$, $q=\pi(D) \pi(C)$. Now let us pick $\delta \in(0,1)$ sufficiently small, depending on the norms of $A, B, C, D$. More precisely, we require $\delta \in(0,1)$ to satisfy

$$
\|A\|\|B\|(1-\delta)^{-1} \delta+\delta<1 / 2, \quad\|C\|\|D\|(1-\delta)^{-1} \delta+\delta<1 / 2
$$

Write $P=\left(p_{n}\right)$ and $Q=\left(q_{n}\right)$, then $p q=0=q p$ is equivalent to $\lim _{n \rightarrow \infty}\left\|p_{n} q_{n}\right\|=$ $0=\lim _{n \rightarrow \infty}\left\|q_{n} p_{n}\right\|$. So there is $M \in \mathbb{N}$ such that $\left\|p_{n} q_{n}\right\|,\left\|q_{n} p_{n}\right\|<\delta$ for every $n \geq M$, and since $p_{n}, q_{n} \in \mathcal{A}_{n}$ are commuting idempotents it follows that $p_{n} q_{n}$ is an idempotent of small norm, so $p_{n} q_{n}=0$; similarly $q_{n} p_{n}=0$.

The aim of the following is to show that the idempotents $p_{n}$ and $q_{n}$ are not only eventually orthogonal, but equivalent to the unit element $1_{n}$ of $\mathcal{A}_{n}$, eventually.

We observe that $1=\pi(A) \pi(B)$ is equivalent to $\lim _{n \rightarrow \infty}\left\|1_{n}-a_{n} b_{n}\right\|=0$, thus there is $M^{\prime} \geq M$ such that $\left\|1_{n}-a_{n} b_{n}\right\|<\delta$ for every $n \geq M^{\prime}$. Consequently, by the Carl Neumann series $u_{n}:=a_{n} b_{n} \in \operatorname{inv}\left(\mathcal{A}_{n}\right),\left\|u_{n}^{-1}\right\|<(1-\delta)^{-1}$ and $\left\|1_{n}-u_{n}^{-1}\right\|<$ $\delta(1-\delta)^{-1}$ for all $n \geq M^{\prime}$. Thus we can define $p_{n}^{\prime}:=b_{n} u_{n}^{-1} a_{n}$ for all $n \geq M^{\prime}$ and it is immediate that $p_{n}^{\prime} \in \mathcal{A}_{n}$ is an idempotent with $p_{n}^{\prime} \sim 1_{n}$. We also have that $\sup _{n \geq M^{\prime}}\left\|p_{n}^{\prime}\right\| \leq\|A\|\|B\|(1-\delta)^{-1}$.

Analogously, there is $M^{\prime \prime} \geq M^{\prime}$ such that $v_{n}:=c_{n} d_{n} \in \operatorname{inv}\left(\mathcal{A}_{n}\right),\left\|v_{n}^{-1}\right\|<(1-\delta)^{-1}$ and $\left\|1_{n}-v_{n}^{-1}\right\|<\delta(1-\delta)^{-1}$ for all $n \geq M^{\prime \prime}$. Then define $q_{n}^{\prime}:=d_{n} v_{n}^{-1} c_{n}$ for all $n \geq M^{\prime \prime}$, so that $q_{n}^{\prime}$ is an idempotent with $q_{n}^{\prime} \sim 1_{n}$, and $\sup _{n \geq M^{\prime \prime}}\left\|q_{n}^{\prime}\right\| \leq\|C\|\|D\|(1-\delta)^{-1}$.

The equality $p=\pi(B) \pi(A)$ is equivalent to saying that $\lim _{n \rightarrow \infty}\left\|p_{n}-b_{n} a_{n}\right\|=0$, and similarly $q=\pi(D) \pi(C)$ is equivalent to $\lim _{n \rightarrow \infty}\left\|q_{n}-d_{n} c_{n}\right\|=0$. So there is $N \geq M^{\prime \prime}$ such that $\left\|p_{n}-b_{n} a_{n}\right\|<\delta$ and $\left\|q_{n}-d_{n} c_{n}\right\|<\delta$ whenever $n \geq N$. 
For every $n \geq N$ we have

$$
\begin{aligned}
\left\|p_{n}^{\prime}-p_{n}\right\| & \leq\left\|b_{n} u_{n}^{-1} a_{n}-b_{n} a_{n}\right\|+\left\|b_{n} a_{n}-p_{n}\right\| \\
& \leq\left\|b_{n}\right\|\left\|u_{n}^{-1}-1_{n}\right\|\left\|a_{n}\right\|+\left\|b_{n} a_{n}-p_{n}\right\| \\
& \leq\|A\|\|B\|(1-\delta)^{-1} \delta+\delta \\
& <1 / 2
\end{aligned}
$$

Therefore by Lemma 2.8 it follows that $p_{n}^{\prime} \sim p_{n}$, and since $\sim$ is an equivalence relation on the set of idempotents of $\mathcal{A}_{n}$, we have $p_{n} \sim 1_{n}$. Similarly, we conclude $q_{n} \sim 1_{n}$ for $n \geq N$. Since $p_{n}$ and $q_{n}$ are orthogonal, the claim follows.

Remark 4.3 We see from the first paragraph of the proof of Theorem 4.2 that pairs of mutually orthogonal idempotents from $\operatorname{Asy}\left(\mathcal{A}_{n}\right)$ can be lifted to mutually orthogonal idempotents in $\ell^{\infty}\left(\mathcal{A}_{n}\right)$. More precisely, if $p, q \in \operatorname{Asy}\left(\mathcal{A}_{n}\right)$ are idempotents with $p q=$ $0=q p$, then there exist idempotents $P, Q=\in \ell^{\infty}\left(\mathcal{A}_{n}\right)$ such that $p=\pi(P), q=\pi(Q)$, and $P Q=0=Q P$.

\subsection{An application to inductive limits of unital Banach algebras}

The construction of inductive limits of unital Banach algebras is given in [2, Section 3.3] and [22, Section 1.3.4], for example. For us it will be enough to use the characterisation in terms of a universal property. Inductive limits seem to be more commonly considered in the setting of $C^{*}$-algebras (where all connecting maps are contractions) or for locally convex spaces. In the general Banach algebra setting there are some subtleties, which we note below.

Let $I$ be a directed set and let $\left(\mathcal{A}_{i}\right)_{i \in I}$ be a family of unital Banach algebras. We suppose that for $i \leq j$ there is a bounded unital homomorphism $\varphi_{j, i}: \mathcal{A}_{i} \rightarrow \mathcal{A}_{j}$, called the compatibility morphism, which satisfies that $\varphi_{i, i}=\operatorname{id}_{\mathcal{A}_{i}}$ for each $i \in I, \varphi_{k, j} \circ \varphi_{j, i}=\varphi_{k, i}$ for $i \leq j \leq k$, and $\lim \sup _{j \geq i}\left\|\varphi_{j, i}\right\|<\infty$ for each $i \in I$. We remark that the construction will still work under the weaker condition that for each $i$ there is $K_{i}$ with $\lim \sup _{j \geq i}\left\|\varphi_{j, i}(a)\right\| \leq K_{i}\|a\|$ for $a \in \mathcal{A}_{i}$. However, this is not equivalent to the stronger condition for general directed sets $I$ : the Uniform Boundedness Principle does not apply, due to the use of lim sup (this is erroneously claimed in [2, 22]; the claim would hold with $I=\mathbb{N}$ ).

The (Banach algebra) inductive limit $\mathcal{A}=\lim \mathcal{A}_{i}$ is uniquely (up to isometric isomorphism) characterised by the universal property that:

(1) for each $i \in I$ there is a bounded unital algebra homomorphism $\varphi_{i}: \mathcal{A}_{i} \rightarrow \mathcal{A}$ with $\left\|\varphi_{i}(a)\right\| \leq \limsup _{j \geq i}\left\|\varphi_{j, i}(a)\right\|$ for $a \in A_{i}$

(2) for $i \leq j$ we have that $\varphi_{i}=\varphi_{j} \circ \varphi_{j, i}$;

(3) if $\mathcal{B}$ is another unital Banach algebra with bounded unital algebra homomorphisms $\phi_{i}: \mathcal{A}_{i} \rightarrow \mathcal{B}$ with $\phi_{i}=\phi_{j} \circ \varphi_{j, i}$ for $i \leq j$, and with $\left\|\phi_{i}(a)\right\| \leq \lim \sup _{j \geq i}\left\|\varphi_{j, i}(a)\right\|$ for each $i \in I, a \in A_{i}$, then there is a unique contractive unital algebra homomorphism $\phi: \mathcal{A} \rightarrow \mathcal{B}$ with $\phi \circ \varphi_{i}=\phi_{i}$ for each $i \in I$. 
These conditions then imply that in (1) we have equality: $\left\|\varphi_{i}(a)\right\|=\lim \sup _{j \geq i}\left\|\varphi_{j, i}(a)\right\|$ for $i \in I$ and $a \in A_{i}$. The universal property, (3), in particular uniqueness of $\phi$, implies that the union of the images of the $\varphi_{i}$ are dense in $\lim _{\longrightarrow} \mathcal{A}_{i}$.

We remark that without the rather explicit norm condition, we do not seem to obtain a universal condition, at this level of generality. If each $\mathcal{A}_{i}$ is a $C^{*}$-algebra with each compatibility morphism a $*$-homomorphism, then $\mathcal{A}$ is a $C^{*}$-algebra, and each compatibility morphism is a contraction. Let now $\mathcal{B}$ be another $C^{*}$-algebra with $*$-homomorphisms $\phi_{i}: \mathcal{A}_{i} \rightarrow \mathcal{B}$ with $\phi_{i}=\phi_{j} \circ \varphi_{j, i}$ for each $i \leq j$. Then for $a \in \mathcal{A}_{i}$ we have $\left\|\phi_{i}(a)\right\|=\left\|\phi_{j}\left(\varphi_{j, i}(a)\right)\right\| \leq\left\|\varphi_{j, i}(a)\right\|$ for each $j \geq i$, and so the norm condition is automatic in this situation.

Proposition 4.4 Let $\left(\left(\mathcal{A}_{n}\right),\left(\varphi_{i, j}\right)\right)$ be an inductive system of unital Banach algebras, indexed by $\mathbb{N}$. There is an isometric unital algebra homomorphism $\theta: \lim _{\longrightarrow} \mathcal{A}_{n} \rightarrow \operatorname{Asy}\left(\mathcal{A}_{n}\right)$.

Proof We use the universal property with $\mathcal{B}=\operatorname{Asy}\left(\mathcal{A}_{n}\right)$. Denote by $\pi: \ell^{\infty}\left(\mathcal{A}_{n}\right) \rightarrow \mathcal{B}$ the natural quotient map. For each $n$ define $\phi_{n}: \mathcal{A}_{n} \rightarrow \mathcal{B}$ by

$$
\phi_{n}(a)=\pi(\underbrace{0,0, \ldots, 0}_{(n-1) \text { terms }}, a, \varphi_{n+1, n}(a), \ldots, \varphi_{i, n}(a), \ldots) .
$$

It is easy to see that the family $\left(\phi_{n}\right)$ satisfies the required commutation relations. Further, by the definition of the norm on $\mathcal{B}$, we have that $\left\|\phi_{n}(a)\right\|=\lim \sup _{k \geq n}\left\|\varphi_{k, n}(a)\right\|$ for all $n \in \mathbb{N}$, which implies the required norm relation. There is hence a unital contractive homomorphism $\theta: \lim _{\longrightarrow} \mathcal{A}_{n} \rightarrow \operatorname{Asy}\left(\mathcal{A}_{n}\right)$ with $\phi_{n}=\theta \circ \varphi_{n}$ for each $n \in \mathbb{N}$. By our remark about condition (1), it follows that $\left\|\phi_{n}(a)\right\|=\left\|\varphi_{n}(a)\right\|$ for each $n \in \mathbb{N}$ and $a \in \mathcal{A}_{n}$. Thus $\theta$ is actually isometric on the image of $\varphi_{n}$, and as the union of such images is dense in $\lim _{\longrightarrow} \mathcal{A}_{n}$, it follows that $\theta$ is isometric, as claimed.

The following lemma is straightforward.

Lemma 4.5 Let $\mathcal{A}, \mathcal{B}$ be unital algebras and let $\psi: \mathcal{A} \rightarrow \mathcal{B}$ be an algebra homomorphism which preserves the unit. If $\mathcal{A}$ is properly infinite, then so is $\mathcal{B}$.

Corollary 4.6 Let $\left(\mathcal{A}_{n}\right)$ be an inductive system of unital Banach algebras. If $\lim \mathcal{A}_{n}$ is properly infinite then there is $N \in \mathbb{N}$ such that $\mathcal{A}_{n}$ is properly infinite for every $n \geq \vec{N}$.

Proof By Proposition 4.4 there is an (contractive) algebra homomorphism $\theta$ : $\lim _{\rightarrow} \mathcal{A}_{n} \rightarrow \operatorname{Asy}\left(\mathcal{A}_{n}\right)$ which preserves the unit, hence by Lemma 4.5 the asymp$\overrightarrow{\text { totic }}$ sequence algebra $\operatorname{Asy}\left(\mathcal{A}_{n}\right)$ is properly infinite. The claim now follows from Theorem 4.2.

Remark 4.7 It is an unpublished observation of James Gabe that for $C^{*}$-algebras, Corollary 4.6 follows from the semiprojectivity of the Cuntz algebra $O_{\infty}$. We would like to thank him for communicating this result to us. 


\subsection{When the sequence consists of properly infinite algebras}

We first demonstrate that the converse of Theorem 4.2 is false in general. For a unital Banach algebra $\mathcal{A}$ we define

$$
C_{\mathrm{PI}}(\mathcal{A}):=\inf \{\|a\|\|b\|\|c\|\|d\|: a, b, c, d \in \mathcal{A}, a b=1=c d, a d=0=c b\}
$$

It will also be useful to define an auxiliary constant

$$
C_{\mathrm{PI}}^{\prime}(\mathcal{A}):=\inf \left\{\|p\|\|q\|: p, q \in \mathcal{A}, p^{2}=p, q^{2}=q, p \sim 1 \sim q, p \perp q\right\} .
$$

Notice that if we have $a, b, c, d$ as in the definition of $C_{\mathrm{PI}}(\mathcal{A})$ then setting $p=b a, q=d c$ then $p^{2}=b a b a=b a=p$ and similarly $q^{2}=q, p q=q p=0$, and $p \sim 1 \sim q$ because $a b=1=c d$. As then $\|p\|\|q\| \leq\|a\|\|b\|\|c\|\|d\|$ we see that $C_{\mathrm{PI}}^{\prime}(\mathcal{A}) \leq C_{\mathrm{PI}}(\mathcal{A})$. If $\mathcal{A}$ is properly infinite then $1 \leq C_{\mathrm{PI}}(\mathcal{A})<+\infty$, otherwise $C_{\mathrm{PI}}(\mathcal{A})=+\infty$. Clearly $C_{\mathrm{PI}}^{\prime}(\mathcal{A})=+\infty$ if and only if $C_{\mathrm{PI}}(\mathcal{A})=+\infty$.

As when we considered an algebra being Dedekind-infinite, the constant $C_{\mathrm{PI}}^{\prime}$ seems more natural, but $C_{\mathrm{PI}}$ seems more useful. However, for being properly infinite, we shall actually obtain a complete characterisation (see Proposition 4.9 and Proposition 4.17) using $C_{\mathrm{PI}}$. Furthermore, Proposition 4.14 shows that $C_{\mathrm{PI}}^{\prime}$ and $C_{\mathrm{PI}}$ are not comparable.

Notice that if $\mathcal{A}$ is properly infinite, then it is Dedekind-infinite, because if $p, q$ are orthogonal with $p \sim 1$ and $q \sim 1$, we cannot have $p=1$.

Lemma 4.8 Let $\mathcal{A}$ be properly infinite. Then $C_{D I}(\mathcal{A}) \leq C_{P I}(\mathcal{A})$ and $C_{D I}^{\prime}(\mathcal{A}) \leq C_{P I}^{\prime}(\mathcal{A})$.

Proof That $C_{\mathrm{DI}}^{\prime}(\mathcal{A}) \leq C_{\mathrm{PI}}^{\prime}(\mathcal{A})$ is clear, given the remark before the lemma. Let $K>C_{\mathrm{PI}}(\mathcal{A})$ so we can find $a, b, c, d \in \mathcal{A}$ with $\|a\|\|b\|\|c\|\|d\| \leq K$ and $a b=c d=$ $1, b a d c=d c b a=0$. Then $b a \neq 1$, and so $C_{\mathrm{DI}}(\mathcal{A}) \leq\|a\|\|b\|$. As $c d=1$ we have that $\|c\|\|d\| \geq 1$ and so $\|a\|\|b\| \leq K$, from which the result follows.

First we prove a slight strengthening of Theorem 4.2.

Proposition 4.9 Let $\left(\mathcal{A}_{n}\right)$ be a sequence of unital Banach algebras such that $\operatorname{Asy}\left(\mathcal{A}_{n}\right)$ is properly infinite. Then there is a $K \geq 1$ and an $N \in \mathbb{N}$ such that $C_{P I}\left(\mathcal{A}_{n}\right) \leq K$ for all $n \geq N$.

Proof The proof is a refinement of the proof of Theorem 4.2. We shall freely use the notation therein, and assume that all the objects have already been defined and the argument is repeated up until and including the inequality $\left\|p_{n}-p_{n}^{\prime}\right\|<1 / 2$ (equation (4.4)), and the analogous inequality $\left\|q_{n}-q_{n}^{\prime}\right\|<1 / 2$ for each $n \geq N$.

By Remark 2.9, we can find $a_{n}^{\prime}, b_{n}^{\prime}, c_{n}^{\prime}, d_{n}^{\prime} \in \mathcal{A}_{n}$ such that $p_{n}=a_{n}^{\prime} b_{n}^{\prime}, p_{n}^{\prime}=b_{n}^{\prime} a_{n}^{\prime}$, $q_{n}=c_{n}^{\prime} d_{n}^{\prime}, q_{n}^{\prime}=d_{n}^{\prime} c_{n}^{\prime}$, and the inequalities

$$
\begin{aligned}
& \left\|a_{n}^{\prime}\right\|\left\|b_{n}^{\prime}\right\| \leq(4 / 3)\left\|p_{n}\right\|^{2}\left(\left\|2 p_{n}-1_{n}\right\|+1 / 2\right)^{2} \leq(4 / 3)\|P\|^{2}(2\|P\|+3 / 2)^{2} \\
& \left\|c_{n}^{\prime}\right\|\left\|d_{n}^{\prime}\right\| \leq(4 / 3)\left\|q_{n}\right\|^{2}\left(\left\|2 q_{n}-1_{n}\right\|+1 / 2\right)^{2} \leq(4 / 3)\|Q\|^{2}(2\|Q\|+3 / 2)^{2}
\end{aligned}
$$

hold for each $n \geq N$. 
Let us define $\tilde{a}_{n}:=a_{n} b_{n}^{\prime}, \tilde{b}_{n}:=a_{n}^{\prime} b_{n} u_{n}^{-1}$, and $\tilde{c}_{n}:=c_{n} d_{n}^{\prime}, \tilde{d}_{n}:=c_{n}^{\prime} d_{n} v_{n}^{-1}$ for each $n \geq N$. Thus we find

$$
\begin{aligned}
& \tilde{a}_{n} \tilde{b}_{n}=a_{n} b_{n}^{\prime} a_{n}^{\prime} b_{n} u_{n}^{-1}=a_{n} p_{n}^{\prime} b_{n} u_{n}^{-1}=a_{n} b_{n} u_{n}^{-1} a_{n} b_{n} u_{n}^{-1}=u_{n} u_{n}^{-1} u_{n} u_{n}^{-1}=1_{n}, \\
& \tilde{b}_{n} \tilde{a}_{n}=a_{n}^{\prime} b_{n} u_{n}^{-1} a_{n} b_{n}^{\prime}=a_{n}^{\prime} p_{n}^{\prime} b_{n}^{\prime}=a_{n}^{\prime} b_{n}^{\prime} a_{n}^{\prime} b_{n}^{\prime}=p_{n} p_{n}=p_{n},
\end{aligned}
$$

and similarly $\tilde{c}_{n} \tilde{d}_{n}=1_{n}, \tilde{d}_{n} \tilde{c}_{n}=q_{n}$ for each $n \geq N$. We also have the estimates

$$
\begin{aligned}
&\left\|\tilde{a}_{n}\right\|\left\|\tilde{b}_{n}\right\| \leq\left\|a_{n}^{\prime}\right\|\left\|b_{n}^{\prime}\right\|\left\|a_{n}\right\|\left\|b_{n}\right\|\left\|u_{n}^{-1}\right\| \leq 4(3-3 \delta)^{-1}\|A\|\|B\|\|P\|^{2}(2\|P\|+3 / 2)^{2}, \\
&\left\|\tilde{c}_{n}\right\|\left\|\tilde{d}_{n}\right\| \leq 4(3-3 \delta)^{-1}\|C\|\|D\|\|Q\|^{2}(2\|Q\|+3 / 2)^{2} .
\end{aligned}
$$

Notice finally that $\tilde{b}_{n} \tilde{a}_{n} \tilde{d}_{n} \tilde{c}_{n}=p_{n} q_{n}=0=q_{n} p_{n}=\tilde{d}_{n} \tilde{c}_{n} \tilde{b}_{n} \tilde{a}_{n}$, and hence $\tilde{a}_{n} \tilde{d}_{n}=$ $0=\tilde{c}_{n} \tilde{b}_{n}$. Recalling that $\delta \in(0,1)$ depends only on the norms of $A, B, C$ and $D$ we conclude $\sup _{n \geq N} C_{\mathrm{PI}}\left(\mathcal{A}_{n}\right)<+\infty$.

We now aim to construct counter-examples to the converse of Theorem 4.2, for which we continue to use semigroup algebras. However, we now need to add a "zero element".

We say that $S$ is a monoid with a zero element if $S$ is a monoid with at least two elements and there exists a $\diamond \in S$ such that $\diamond s=\diamond=s \diamond$ for all $s \in S$. If such a $\diamond \in S$ exists then it is necessarily unique. As we assume that $S$ has more than one element, we have that $\diamond$ is different from the multiplicative identity $e \in S$.

Let $\omega: S \rightarrow(0,+\infty)$ be a weight on $S$. Let $\mu:=\left.\omega\right|_{S \backslash\{\diamond\}}$, then $\mu: S \backslash\{\diamond\} \rightarrow(0,+\infty)$ is such that $\mu(e)=1$. Every $\mu: S \backslash\{\diamond\} \rightarrow(0,+\infty)$ arising in this way, as a restriction of a weight, will be referred to as a quasi-weight.

We now explain how to define a product on the Banach space $\ell^{1}(S \backslash\{\diamond\}, \mu)$ (see also [4, Section 3.2] for a similar treatment). This is accomplished by identifying $\ell^{1}(S \backslash\{\diamond\}, \mu)$ with the quotient algebra $\ell^{1}(S, \omega) / \mathbb{C} \delta_{\diamond}$. With more details, we first notice that $\mathbb{C} \delta_{\diamond}$ is a closed two-sided ideal in $\left(\ell^{1}(S, \omega), *\right)$. Let $\pi: \ell^{1}(S, \omega) \rightarrow \ell^{1}(S, \omega) / \mathbb{C} \delta_{\diamond}$ denote the quotient map. The symbol · will stand for the product on $\ell^{1}(S, \omega) / \mathbb{C} \delta_{\diamond}$ induced by $*$. Let us consider the restriction map

$$
\psi: \ell^{1}(S, \omega) \rightarrow \ell^{1}(S \backslash\{\diamond\}, \mu) ;\left.\quad f \mapsto f\right|_{S \backslash\{\diamond\}}
$$

This is a linear contractive surjection with $\operatorname{Ker}(\psi)=\mathbb{C} \delta_{\diamond}$. Moreover, it also immediately follows from the definition that $\psi$ maps the open unit ball of $\ell^{1}(S, \omega)$ onto the open unit ball of $\ell^{1}(S \backslash\{\diamond\}, \mu)$. Consequently, there is an isometric linear bijection

$$
\varphi: \ell^{1}(S, \omega) / \mathbb{C} \delta_{\diamond} \rightarrow \ell^{1}(S \backslash\{\diamond\}, \mu)
$$

which satisfies $\varphi \circ \pi=\psi$. This allows us to define a product on $\ell^{1}(S \backslash\{\diamond\}, \mu)$ by setting

$$
f \# g:=\varphi\left(\varphi^{-1}(f) \cdot \varphi^{-1}(g)\right) \quad\left(f, g \in \ell^{1}(S \backslash\{\diamond\}, \mu)\right) .
$$

It is elementary to see that \# is an algebra product on $\left.\ell^{1}(S \backslash\{\diamond\}, \mu)\right)$. Furthermore, $\left(\ell^{1}(S \backslash\right.$ $\{\diamond\}, \mu), \#)$ is a Banach algebra since $\|f \# g\|_{\mu} \leq\|f\|_{\mu}\|g\|_{\mu}$ holds for all $f, g \in \ell^{1}(S \backslash$ $\{\diamond\}, \mu)$ as the map $\varphi$ is an isometry. 
For our purposes the most important property of $\ell^{1}(S \backslash\{\diamond\}, \mu)$ is that for every $s, t \in S \backslash\{\diamond\}$,

$$
\delta_{s} \# \delta_{t}= \begin{cases}\delta_{s t} & \text { if } s t \neq \diamond \\ 0 & \text { if } s t=\diamond .\end{cases}
$$

The above equality holds for the following reason. Observe that for $r \in S \backslash\{\diamond\}$, we simply have $\delta_{r}=\left.\delta_{r}\right|_{S \backslash\{\diamond\}}=\psi\left(\delta_{r}\right)$. Consequently whenever $s, t \in S \backslash\{\diamond\}$ then

$$
\begin{aligned}
\varphi^{-1}\left(\delta_{s} \# \delta_{t}\right) & =\varphi^{-1}\left(\delta_{s}\right) \cdot \varphi^{-1}\left(\delta_{t}\right)=\varphi^{-1}\left(\psi\left(\delta_{s}\right)\right) \cdot \varphi^{-1}\left(\psi\left(\delta_{t}\right)\right)=\pi\left(\delta_{s}\right) \cdot \pi\left(\delta_{t}\right) \\
& =\pi\left(\delta_{s} * \delta_{t}\right)=\pi\left(\delta_{s t}\right)=\varphi^{-1}\left(\psi\left(\delta_{s t}\right)\right) .
\end{aligned}
$$

On the one hand if $s t=\diamond$ then $\psi\left(\delta_{s t}\right)=\psi\left(\delta_{\diamond}\right)=0$. On the other hand if $s t \neq \diamond$ then $\psi\left(\delta_{s t}\right)=\delta_{s t}$, thus proving the claim.

In particular it follows from equation (4.13) that $\left(\ell^{1}(S \backslash\{\diamond\}, \mu)\right.$,\#) is a unital Banach algebra with $\delta_{e}$ being the unit, and such that $\left\|\delta_{e}\right\|_{\mu}=\mu(e)=1$. The proof of the following is entirely analogous to that of Proposition 3.6.

Proposition 4.10 Let $S$ be a monoid with multiplicative identity $e \in S$ and a zero element $\diamond \in S$. Let $\mu: S \backslash\{\diamond\} \rightarrow[1,+\infty)$ be a quasi-weight on $S \backslash\{\diamond\}$. Let $p \in\left(\ell^{1}(S \backslash\{\diamond\}, \mu), \#\right)$ be a non-zero idempotent such that $p \neq \delta_{e}$. Then

$$
\|p\|_{\mu} \geq \frac{1}{2} \inf \{\mu(s): s \in S, s \neq e, s \neq \diamond\} .
$$

In the following $\mathrm{Cu}_{2}$ denotes the second Cuntz semigroup

$$
\left\langle a_{1}, a_{2}, b_{1}, b_{2}: a_{1} b_{1}=e=a_{2} b_{2}, a_{1} b_{2}=\diamond=a_{2} b_{1}\right\rangle,
$$

as defined in, for example, [24, Page 141, Definition 2.2]. Here $e \in \mathrm{Cu}_{2}$ and $\diamond \in \mathrm{Cu}_{2}$ denote the multiplicative identity and the zero element, respectively, rendering $\mathrm{Cu}_{2}$ a monoid with a zero element.

Fix $n \in \mathbb{N}$. Let $\mu_{n}: \mathrm{Cu}_{2} \backslash\{\diamond\} \rightarrow[1,+\infty)$ be a quasi-weight on $\mathrm{Cu}_{2} \backslash\{\diamond\}$ defined as $\mu_{n}(e)=1$ and $\mu_{n}(s)=n$ whenever $s \in \mathrm{Cu}_{2} \backslash\{e, \diamond\}$. Notice that this arises from the weight $\omega_{n}: \mathrm{Cu}_{2} \rightarrow[1,+\infty)$ defined by $\omega_{n}(e)=\omega_{n}(\diamond)=1$ and $\omega_{n}(s)=n$ otherwise.

Theorem 4.11 Let $\mathcal{A}_{n}:=\left(\ell^{1}\left(C u_{2} \backslash\{\diamond\}, \mu_{n}\right), \#\right)$ for every $n \in \mathbb{N}$. Then $\left(\mathcal{A}_{n}\right)$ is a sequence of properly infinite Banach algebras such that $\operatorname{Asy}\left(\mathcal{A}_{n}\right)$ is not properly infinite.

Proof Fix $n \in \mathbb{N}$. Let $p:=\delta_{b_{1}} \# \delta_{a_{1}}$ and $q:=\delta_{b_{2}} \# \delta_{a_{2}}$. Then $p, q \in \mathcal{A}_{n}$ are idempotents with $p \sim \delta_{e} \sim q$ and $p \perp q$ plainly because of the defining properties of $\mathrm{Cu}_{2}$ and equation (4.13). This in particular shows that $\mathcal{A}_{n}$ is properly infinite.

Let $p, q \in \mathcal{A}_{n}$ be arbitrary idempotents satisfying $p \sim \delta_{e} \sim q$ and $p \perp q$. Clearly $p, q \notin\left\{\delta_{e}, 0\right\}$, hence Proposition 4.10 yields $\|p\|_{\mu_{n}},\|q\|_{\mu_{n}} \geq n / 2$, and consequently $C_{\mathrm{PI}}^{\prime}\left(\mathcal{A}_{n}\right) \geq n^{2} / 4$. In view of Proposition 4.9 the Banach algebra $\operatorname{Asy}\left(\mathcal{A}_{n}\right)$ cannot be properly infinite.

Any reduced word in $\mathrm{Cu}_{2}$ is of the form $s=t_{b} s_{a}$, where $s_{a}$ is a word in $a_{1}, a_{2}$ (which are free, so generating a copy of $\mathbb{S}_{2}$ the free semigroup on two generators), and $t_{b}$ is a 
word in $b_{1}, b_{2}$. Consider how to cancel a word of the form $s_{a} t_{b}$. This will be equal to $\diamond$ unless $s_{a} t_{b}=\cdots a_{1}^{n_{3}} a_{2}^{n_{2}} a_{1}^{n_{1}} b_{1}^{n_{1}} b_{2}^{n_{2}} b_{1}^{n_{3}} \cdots$ with perhaps one of $s_{a}$ or $t_{b}$ having extra, unbalanced, terms on the left, or right, respectively. We can express this more succinctly as follows. Define $*$ to be the unique involution on $\mathrm{Cu}_{2}$ with $a_{i}^{*}=b_{i}$ for $i=1,2$ and $e^{*}=e, \diamond^{*}=\diamond$. Notice that $r_{b}^{*} r_{b}=e$ for any word $r_{b}$. Then $s_{a} t_{b}=\diamond$ unless either $s_{a}=r_{a} t_{b}^{*}$ or $t_{b}=s_{a}^{*} r_{b}$, for some words $r_{a}$ and $r_{b}$.

From this, we can see that the idempotents in $\mathrm{Cu}_{2}$ are of the form $s_{b} s_{b}^{*}$ for an arbitrary word $s_{b} \in \mathbb{S}_{2}$. Let $I\left(\mathrm{Cu}_{2}\right)$ be the set of idempotents, excluding $\diamond$. One can also show that if $s \in I\left(\mathrm{Cu}_{2}\right), t \notin I\left(\mathrm{Cu}_{2}\right)$ then st, $t s \notin I\left(\mathrm{Cu}_{2}\right)$. How idempotents multiply is a little more complicated. Let $s=s_{b} s_{b}^{*}, t=t_{b} t_{b}^{*}$, and consider $s t$. This will equal $\diamond$ unless either:

- $t_{b}=s_{b} r_{b}$, in which case $s t=s_{b} s_{b}^{*} s_{b} r_{b} r_{b}^{*} s_{b}^{*}=s_{b} r_{b} r_{b}^{*} s_{b}^{*}=t$; or

- $s_{b}^{*}=r_{a} t_{b}^{*}$, that is, $s_{b}=t_{b} r_{b}$ for some $r_{b}$, in which case

$s t=t_{b} r_{b} r_{b}^{*} t_{b}^{*} t_{b} t_{b}^{*}=t_{b} r_{b} r_{b}^{*} t_{b}^{*}=s$.

This motivates defining $s_{b} \leq t_{b}$ if $t_{b}=s_{b} r_{b}$ for some word $r_{b}$, that is, $s_{b}$ is a prefix of $t_{b}$. Then $s t=t$ when $s_{b} \leq t_{b}$, and $s t=s$ when $t_{b} \leq s_{b}$, and $s t=\diamond$ otherwise.

We can consider $\left(\ell^{1}\left(I\left(\mathrm{Cu}_{2}\right)\right)\right.$,\#). To ease notation, let $\mathbb{S}_{2}(b)$ be the set of words in $b_{1}, b_{2}$, with $\emptyset$ the empty word (the identity), so that a member of $I\left(\mathrm{Cu}_{2}\right)$ has the form $x x^{*}$ for some $x \in \mathbb{S}_{2}(b)$. Let $e_{x}=\delta_{x x^{*}}$, so $\left(e_{x}\right)$ is a basis for $\ell^{1}\left(I\left(\mathrm{Cu}_{2}\right)\right)$ and the product is

$$
e_{x} \# e_{y}= \begin{cases}e_{x} & \text { if } y \leq x \\ e_{y} & \text { if } x \leq y \\ 0 & \text { otherwise }\end{cases}
$$

Lemma 4.12 Let $\mathcal{A}=\left(\ell^{1}\left(I\left(C u_{2}\right)\right)\right.$, \#) and let $f=\sum_{x} f\left(x x^{*}\right) e_{x} \in \mathcal{A}$. Then $f$ is an idempotent if and only if:

(1) $f\left(x x^{*}\right) \in\{-1,0,1\}$ for all $x$, and only finitely many are non-zero;

(2) $\sum_{y<x} f\left(y y^{*}\right) \in\{0,1\}$ for each $x$.

Proof We have that $e_{x} \# e_{y}=e_{z}$ exactly when one of $x, y$ is equal to $z$, and the other is a prefix of $z$. Throughout the rest of the proof we shall write $f(x)$ instead of $f\left(x x^{*}\right)$ for the sake of readibility. Thus

$$
f \# f=\sum_{x, y} f(x) f(y)\left(e_{x} \# e_{y}\right)=\sum_{z}\left(\sum_{x<z} f(z) f(x)+\sum_{x<z} f(x) f(z)+f(z)^{2}\right) e_{z} .
$$

Thus $f \# f=f$ if and only if

$$
f(z)=f(z)^{2}+2 f(z) \sum_{x<z} f(x) \quad\left(z \in \mathbb{S}_{2}(b)\right) .
$$

If the two conditions hold, suppose $\sum_{x<z} f(x)=0$. Let $w=z b_{1}$ (say) so $y<w$ exactly when $y=z$ or $y<z$. Thus $\sum_{y<w} f(y)=f(z)+\sum_{x<z} f(x)=f(z)$ so $f(z) \in$ $\{0,1\}$, and hence (4.17) holds. If $\sum_{x<z} f(x)=1$ then similar reasoning shows that $f(z)+$ $1 \in\{0,1\}$ so $f(z) \neq 1$, and hence (4.17) holds. 
Conversely, we perform induction on the length of the word in $\mathbb{S}_{2}(b)$, again using that if $z$ is a word of length $n+1$ then $z=y b_{i}$ say, for $y$ a word of length $n$, and then $x<z$ if and only if $x=y$ or $x<y$. The details are the same as in the proof of Lemma 3.10.

Proposition 4.13 Let $\mu$ be a quasi-weight on $C u_{2} \backslash\{\diamond\}$ such that $\mu \geq 1$ and $\mu(s) \geq N$ for each $s \notin I\left(C u_{2}\right)$. Then $C_{P I}\left(\ell^{1}\left(C u_{2} \backslash\{\diamond\}, \mu\right), \#\right) \geq(N / 86)^{1 / 3}$

Proof Set $\mathcal{A}=\left(\ell^{1}\left(\mathrm{Cu}_{2} \backslash\{\diamond\}, \mu\right)\right.$. By Lemma 4.8 it suffices to prove that $C_{D I}(\mathcal{A}) \geq$ $(N / 86)^{1 / 3}$. To show this, we can follow almost exactly the strategy of the proof of Proposition 3.11, given the preliminary observations made above.

Proposition 4.14 For each $n \geq 1$ there exists a quasi-weight $\mu_{n}$ on $C u_{2} \backslash\{\diamond\}$ so that with $\mathcal{A}=\left(\ell^{1}\left(C u_{2} \backslash\{\diamond\}, \mu_{n}\right), \#\right)$, we have $C_{P I}^{\prime}(\mathcal{A})=1$ and yet $C_{P I}\left(\mathcal{A}_{n}\right) \geq n$.

Proof We follow the strategy of the proof of Proposition 3.12. Choose $N$ so that $(N / 86)^{1 / 3} \geq n$. The set $X=\left\{e, \diamond, b_{1} a_{1}, b_{2} a_{2}\right\}$ is a sub-semigroup of $\mathrm{Cu}_{2}$, and so the map $\omega: \mathrm{Cu}_{2} \rightarrow[1, \infty)$ defined by $\omega(s)=1$ for $s \in X$ and $\omega(s)=N \in \mathbb{N}$ otherwise, is a weight. Let $\mu_{n}$ be the induced quasi-weight on $\mathrm{Cu}_{2} \backslash\{\diamond\}$. With $p=\delta_{b_{1} a_{1}}$ and $q=\delta_{b_{2} a_{2}}$, we see that $C_{\mathrm{PI}}^{\prime}(\mathcal{A})=1$. However, our quasi-weight satisfies the hypotheses of Proposition 4.13, and so $C_{\mathrm{PI}}(\mathcal{A}) \geq(N / 86)^{1 / 3} \geq n$.

We can prove some similar renorming results. The following is shown in exactly the same way as Proposition 3.16, as if we have orthogonal idemopotents $p, q$ then $\{0,1, p, q\}$ is a (bounded) semigroup in $\mathcal{A}$.

Proposition 4.15 Let $\mathcal{A}$ be a properly infinite Banach algebra. There is an equivalent norm $\|\cdot\|_{0}$ on $\mathcal{A}$ such that $\left(\mathcal{A},\|\cdot\|_{0}\right)$ is a unital Banach algebra, and $C_{P I}^{\prime}\left(\mathcal{A},\|\cdot\|_{0}\right)=1$.

Proposition 4.16 For each $K>0$ there is a Banach algebra $\mathcal{A}$ and $a, b, c, d \in \mathcal{A}$ with $a b=c d=1, c b=a d=0$, such that, if $\|\cdot\|_{0}$ is any equivalent norm on $\mathcal{A}$, then $\|a\|_{0}\|b\|_{0}\|c\|_{0}\|d\|_{0} \geq K$.

Proof We follow the strategy of the proof of Proposition 3.18. We have the wordlength $\ell$ on $\mathrm{Cu}_{2}$, where $\ell(\diamond)=0$, and again this is sub-additive. Thus, for $x>0$, the function $\omega_{x}(s)=\exp (x \ell(s))$ is a weight. Let $\mu$ be the quasi-weight given by $\omega$, and set $\mathcal{A}=\left(\left(\ell^{1}\left(\mathrm{Cu}_{2} \backslash\{\diamond\}, \mu_{n}\right), \#\right)\right.$. Let $\|\cdot\|_{0}$ be an equivalent norm on $\mathcal{A}$, say with $m^{-1}\|f\|_{0} \leq$ $\|f\|_{\omega_{x}}$ for each $f \in \mathcal{A}$.

Set $a=\delta_{a_{1}}, b=\delta_{b_{1}}$ and $c=\delta_{a_{2}}, d=\delta_{b_{2}}$. The same argument as used in the proof of Proposition 3.18 now shows that $\|a\|_{0},\|b\|_{0},\|c\|_{0},\|d\|_{0} \geq e^{x}$, which completes the proof.

Again, we leave open the question of whether it is possible to find an absolute constant $K \geq 1$ such that for every properly infinite Banach algebra $\mathcal{A}$ there is an equivalent norm $\|\cdot\|_{0}$ on $\mathcal{A}$ with $C_{\mathrm{PI}}\left(\mathcal{A},\|\cdot\|_{0}\right) \leq K$. 


\subsection{When we have norm control}

As in the Dedekind-finite case, the converse to Theorem 4.2 holds provided we have uniform norm control. Notice that this, when combined with Proposition 4.9, gives a complete characterisation of when $\operatorname{Asy}\left(\mathcal{A}_{n}\right)$ is properly infinite.

Proposition 4.17 Let $\left(\mathcal{A}_{n}\right)$ be a sequence of unital Banach algebras such that $\lim \sup _{n \rightarrow \infty} C_{P I}\left(\mathcal{A}_{n}\right)<\infty$. Then $\operatorname{Asy}\left(\mathcal{A}_{n}\right)$ is properly infinite.

We remark that this hypothesis is weaker than $\sup _{n} C_{\mathrm{PI}}\left(\mathcal{A}_{n}\right)<\infty$, as the hypothesis of the proposition allows finitely many of the $\mathcal{A}_{n}$ to not be properly infinite.

Proof By hypothesis, there are $K>0$ and $N \in \mathbb{N}$ such that for $n \geq N$ we can find $a_{n}, b_{n}, c_{n}, d_{n} \in \mathcal{A}_{n}$ with $\left\|a_{n}\right\|\left\|b_{n}\right\|\left\|c_{n}\right\|\left\|d_{n}\right\| \leq K$ and so that, with $p_{n}=b_{n} a_{n}, q_{n}=$ $d_{n} c_{n}$, we have that $p_{n}, q_{n}$ are mutually orthogonal idempotents with $p_{n} \sim 1 \sim q_{n}$. Notice that by rescaling, we may suppose that $\left\|a_{n}\right\|=\left\|b_{n}\right\|$ and $\left\|c_{n}\right\|=\left\|d_{n}\right\|$. As $a_{n} b_{n}=1$, it follows that $\left\|a_{n}\right\| \geq 1$; similarly $\left\|c_{n}\right\| \geq 1$. Then $\left\|a_{n}\right\|^{2}\left\|c_{n}\right\|^{2} \leq K$ and so $\left\|a_{n}\right\|^{2} \leq K$ and $\left\|c_{n}\right\|^{2} \leq K$.

For $n<N$ define $a_{n}=b_{n}=c_{n}=d_{n}=0$. Then $A=\left(a_{n}\right) \in \ell^{\infty}\left(\mathcal{A}_{n}\right)$ with $\|A\|^{2} \leq K$, and similarly for $B=\left(b_{n}\right), C=\left(c_{n}\right)$ and $D=\left(d_{n}\right)$. We now see that $\pi(A) \pi(B)=1$ in Asy $\left(\mathcal{A}_{n}\right)$, and similarly $\pi(C) \pi(D)=1$. Furthermore, $p=\pi(B) \pi(A)$ and $q=\pi(D) \pi(C)$ are idempotents with $p q=q p=0$. Thus $p \sim 1 \sim q$ and $p, q$ are orthogonal, and so $\operatorname{Asy}\left(\mathcal{A}_{n}\right)$ is properly infinite, as claimed.

Corollary 4.18 Let $\left(\mathcal{A}_{n}\right)$ be a sequence of unital Banach algebras such that there is an $N \in \mathbb{N}$ such that $\mathcal{A}_{n}$ is properly infinite for all $n \geq N$. Moreover, suppose that one of the following two conditions hold:

(1) $\mathcal{A}_{n}=\mathcal{A}_{m}$ for every $n, m \geq N$;

(2) $\mathcal{A}_{n}$ is a $C^{*}$-algebra for each $n \in \mathbb{N}$.

Then $\operatorname{Asy}\left(\mathcal{A}_{n}\right)$ is properly infinite.

Proof When $\mathcal{A}_{n}=\mathcal{A}_{m}$ for $n, m \geq N$, this follows immediately from the preceding result. Now suppose that each $\mathcal{A}_{n}$ is a $C^{*}$-algebra. From Proposition 2.4, a $C^{*}$-algebra $\mathcal{B}$ is properly infinite if and only if there are projections $p, q \in \mathcal{B}$ with $p q=0$ (so also $q p=0$ ) and with $p \approx 1 \approx q$. In particular, $C_{\mathrm{PI}}(\mathcal{B})=1$; and so the result follows again from the previous result.

\subsection{For ultraproducts}

All of these results hold for ultraproducts with suitable modifications. For example, the analogue of combining Propositions 4.9 and 4.17 is the following.

Theorem 4.19 Let $\left(\mathcal{A}_{n}\right)$ be a sequence of Banach algebras, and let $\mathcal{U}$ be an ultrafilter. Then $\left(\mathcal{A}_{n}\right) \mathcal{U}$ is properly infinite if and only if there is $K>0$ such that $\left\{n \in \mathbb{N}: C_{P I}\left(\mathcal{A}_{n}\right) \leq K\right\} \in$ $\mathcal{U}$ 
We remark that again Łośs Theorem could be used. However, here the details of the analogue of Lemma 3.21 seem complex, and we have chosen not to give them.

\section{Stable rank one}

We say that a unital Banach algebra $\mathcal{A}$ has stable rank one if the group of invertible elements $\operatorname{inv}(\mathcal{A})$ is dense in $\mathcal{A}$. We recall, [25, Proposition 3.1], that this is equivalent to either the left, or the right, topological stable rank of $\mathcal{A}$ being 1 . We recall, see [17, Lemma 2.1] for example, that having stable rank one implies being Dedekind finite. As shown in [17, Example 2.2] the converse does not hold. We hence view having stable rank one as a strict strengthening of being Dedekind-finite; and a strengthening which is much studied for $C^{*}$-algebras.

Lemma 5.1 Let $\left(\mathcal{A}_{n}\right)$ be a sequence of unital Banach algebras. Then

$$
\operatorname{inv}\left(\operatorname{Asy}\left(\mathcal{A}_{n}\right)\right)=\pi\left(\operatorname{inv}\left(\ell^{\infty}\left(\mathcal{A}_{n}\right)\right)\right) .
$$

Proof For the non-trivial direction, let us pick an arbitrary $A=\left(a_{n}\right) \in \ell^{\infty}\left(\mathcal{A}_{n}\right)$ with $\pi(A) \in \operatorname{inv}\left(\operatorname{Asy}\left(\mathcal{A}_{n}\right)\right)$. Thus there is $C=\left(c_{n}\right) \in \ell^{\infty}\left(\mathcal{A}_{n}\right)$ with $\pi(A) \pi(C)=\pi(C) \pi(A)=$ 1 , that is, with

$$
\lim _{n \rightarrow \infty}\left\|c_{n} a_{n}-1_{n}\right\|=\lim _{n \rightarrow \infty}\left\|a_{n} c_{n}-1_{n}\right\|=0 .
$$

Set $u_{n}=c_{n} a_{n}$ and $v_{n}=a_{n} c_{n}$ for each $n$, so there is $N$ with $\left\|u_{n}-1_{n}\right\|<1 / 2$ and $\left\|v_{n}-1_{n}\right\|<1 / 2$ for $n \geq N$. Consequently, for $n \geq N$ we have that $u_{n}, v_{n} \in \operatorname{inv}\left(\mathcal{A}_{n}\right)$ with $\left\|u_{n}^{-1}\right\|,\left\|v_{n}^{-1}\right\| \leq 2$. As $a_{n} u_{n}=a_{n} c_{n} a_{n}=v_{n} a_{n}$ for each $n$, we have that $a_{n} u_{n}^{-1}=$ $v_{n}^{-1} a_{n}$ for $n \geq N$. Observe that

$$
a_{n}\left(c_{n} v_{n}^{-1}\right)=1_{n}, \quad\left(c_{n} v_{n}^{-1}\right) a_{n}=c_{n} a_{n} u_{n}^{-1}=1_{n},
$$

and so $a_{n} \in \operatorname{inv}\left(\mathcal{A}_{n}\right)$ with $a_{n}^{-1}=c_{n} v_{n}^{-1}$ and hence $\left\|a_{n}^{-1}\right\| \leq 2\|C\|$. Define

$$
a_{n}^{\prime}=\left\{\begin{array}{ll}
a_{n} & \text { if } n \geq N, \\
1_{n} & \text { otherwise, }
\end{array} \quad b_{n}= \begin{cases}a_{n}^{-1} & \text { if } n \geq N, \\
1_{n} & \text { otherwise. }\end{cases}\right.
$$

Let $A^{\prime}=\left(a_{n}^{\prime}\right), B=\left(b_{n}\right)$ so that $A^{\prime}, B \in \ell^{\infty}\left(\mathcal{A}_{n}\right)$ and $A^{\prime} B=B A^{\prime}=1$, so that $A^{\prime} \in$ $\operatorname{inv}\left(\ell^{\infty}\left(\mathcal{A}_{n}\right)\right)$. As $\pi(A)=\pi\left(A^{\prime}\right)$ the claimed result follows.

Proposition 5.2 Let $\mathcal{A}$ be a unital Banach algebra such that $\operatorname{Asy}(\mathcal{A})$ has stable rank one. Then also $\mathcal{A}$ has stable rank one.

Proof If not, then there is $a \in \mathcal{A}$ and $\epsilon>0$ with $\|a-b\| \geq \epsilon$ for each $b \in \operatorname{inv}(\mathcal{A})$. Let $A=(a) \in \ell^{\infty}(\mathcal{A})$. Since $\operatorname{Asy}(\mathcal{A})$ has stable rank one, there is $c \in \operatorname{inv}(\operatorname{Asy}(\mathcal{A}))$ with $\|\pi(A)-c\|<\epsilon / 2$. By Lemma 5.1 there is $C=\left(c_{n}\right) \in \operatorname{inv}\left(\ell^{\infty}(\mathcal{A})\right)$ with $\pi(C)=c$, so that

$$
\epsilon / 2>\|\pi(A)-c\|=\|\pi(A)-\pi(C)\|=\limsup _{n \rightarrow \infty}\left\|a-c_{n}\right\|
$$

Hence there is some $n \in \mathbb{N}$ with $\left\|a-c_{n}\right\|<\epsilon$, and as each $c_{n}$ is invertible, this gives the required contradiction. 
We now wish to improve this result, and completely characterise when $\operatorname{Asy}(\mathcal{A})$ has stable rank one in terms of "uniform" approximation by invertibles for $\mathcal{A}$. We give below, in Theorem 5.10, a counter-example to show that $\mathcal{A}$ can have stable rank one while $\operatorname{Asy}(\mathcal{A})$ does not. For $C^{*}$-algebras, this does always hold, see Proposition 5.8.

Proposition 5.3 Let $\mathcal{A}$ be a unital Banach algebra. The following are equivalent:

(1) There is a function $f:(0, \infty) \rightarrow \mathbb{R}$ such that for $\epsilon>0$ and $a \in \mathcal{A}$ with $\|a\| \leq 1$ there is $b \in \operatorname{inv}(\mathcal{A})$ with $\|a-b\|<\epsilon$ and $\left\|b^{-1}\right\| \leq f(\epsilon)$;

(2) $\ell^{\infty}(\mathcal{A})$ has stable rank one;

(3) $\operatorname{Asy}(\mathcal{A})$ has stable rank one.

Proof Suppose $f$ exists. Let $A=\left(a_{n}\right) \in \ell^{\infty}(\mathcal{A})$. By homogeneity we may suppose that $\left\|a_{n}\right\| \leq 1$ for each $n$. Given $\epsilon>0$, for each $n$ we can find $b_{n} \in \operatorname{inv}(\mathcal{A})$ with $\left\|a_{n}-b_{n}\right\|<\epsilon$ and $\left\|b_{n}^{-1}\right\| \leq f(\epsilon)$. Thus $B=\left(b_{n}\right) \in \ell^{\infty}(\mathcal{A})$ and $\left(b_{n}^{-1}\right)$ is also in $\ell^{\infty}(\mathcal{A})$. So $B \in \operatorname{inv}\left(\ell^{\infty}(\mathcal{A})\right)$ and $\|A-B\| \leq \epsilon$. As $\epsilon>0$ and $A$ were arbitrary, this shows that $\ell^{\infty}(\mathcal{A})$ has stable rank one.

If $\ell^{\infty}(\mathcal{A})$ has stable rank one, also $\operatorname{Asy}(\mathcal{A})$ has stable rank one.

Now suppose that $\operatorname{Asy}(\mathcal{A})$ has stable rank one. For $\epsilon>0$ and $a \in \mathcal{A}$ with $\|a\| \leq 1$ let $I_{a}^{\epsilon}=\{b \in \operatorname{inv}(\mathcal{A}):\|a-b\|<\epsilon\}$. That $f$ exists is equivalent to showing that for each $\epsilon>0$,

$$
\sup \left\{\inf \left\{\left\|b^{-1}\right\|: b \in I_{a}^{\epsilon}\right\}:\|a\| \leq 1\right\}<\infty .
$$

Suppose this is not so. Then there is $\epsilon>0$ and a sequence $\left(a_{n}\right)$ with $\left\|a_{n}\right\| \leq 1$ for each $n \in \mathbb{N}$, and with $\left\|b^{-1}\right\| \geq n$ for each $b \in I_{a_{n}}^{\epsilon}$. Let $A:=\left(a_{n}\right) \in \ell^{\infty}(\mathcal{A})$ and $a:=\pi(A) \in \operatorname{Asy}(\mathcal{A})$, so there is $c \in \operatorname{inv}(\operatorname{Asy}(\mathcal{A}))$ with $\|a-c\|<\epsilon / 2$. Again, we can find $C=\left(c_{n}\right) \in \operatorname{inv}\left(\ell^{\infty}(\mathcal{A})\right)$ with $c=\pi(C)$. Thus in particular, there is $M>0$ with $\left\|c_{n}^{-1}\right\| \leq M$ for all $n \in \mathbb{N}$. As $\lim _{\sup _{n \rightarrow \infty}}\left\|a_{n}-c_{n}\right\|<\epsilon / 2$, there is $N \in \mathbb{N}$ such that $\left\|a_{n}-c_{n}\right\|<\epsilon / 2$ and hence $c_{n} \in I_{a_{n}}^{\epsilon}$ for each $n \geq N$. Then for any $n>\max (M, N)$ we obtain $\left\|c_{n}^{-1}\right\| \leq M \leq \max (M, N)<n \leq\left\|c_{n}^{-1}\right\|$, a contradiction.

We remark that it seems somewhat harder to characterise when $\operatorname{Asy}\left(\mathcal{A}_{n}\right)$ has stable rank one, for a sequence $\left(\mathcal{A}_{n}\right)$ of varying Banach algebras. In the next section we develop some results which allow us to say something about this more general situation.

\subsection{Stable rank one as a "three space property"}

Having stable rank one is not a three-space property (see [25, Examples 4.13]), but in our special situation we can say something. The following is the Banach-algebraic analogue of the ring-theoretic lemma [17, Lemma 2.10]. We recall that if $\mathcal{A}$ is a unital algebra over a field $K$ with multiplicative identity $1_{\mathcal{A}}$, and $\mathcal{J} \unlhd \mathcal{A}$ is a two-sided ideal, then $\tilde{\mathcal{J}}$ denotes the unital subalgebra $K 1_{\mathcal{A}}+\mathcal{J}$. Moreover, $\operatorname{inv}(\tilde{\mathcal{J}})=\operatorname{inv}(\mathcal{A}) \cap \tilde{\mathcal{J}}$ (see $[17$, Lemma 2.4]).

Proposition 5.4 Let $\mathcal{A}$ be a unital Banach algebra and let $\mathcal{J} \unlhd \mathcal{A}$ be a closed two-sided ideal such that both $\tilde{\mathcal{J}}$ and $\mathcal{A} / \mathcal{J}$ have stable rank one. Let $\pi: \mathcal{A} \rightarrow \mathcal{A} / \mathcal{J}$ denote the quotient map. If $\pi(\operatorname{inv}(\mathcal{A}))=\operatorname{inv}(\mathcal{A} / \mathcal{J})$ then $\mathcal{A}$ has stable rank one. 
Proof Let $a \in \mathcal{A}$ and $\epsilon>0$ be arbitrary. Since $\mathcal{A} / \mathcal{J}$ has stable rank one, there is $c \in \mathcal{A}$ such that $\pi(c) \in \operatorname{inv}(\mathcal{A} / \mathcal{J})$ and $\|\pi(a)-\pi(c)\|<\epsilon / 2$. By the assumption there is $d \in \operatorname{inv}(\mathcal{A})$ such that $\pi(d)=\pi(c)$ and thus $\|\pi(a)-\pi(d)\|<\epsilon / 2$. Consequently there is $b \in \mathcal{J}$ such that $\|a-d-b\|<\epsilon / 2$. Let us define $a^{\prime}:=b+d$. We observe that

$$
\pi\left(a^{\prime} d^{-1}\right)=\pi\left(b d^{-1}+1\right)=\pi(b) \pi\left(d^{-1}\right)+\pi(1)=\pi(1)
$$

equivalently, $1-a^{\prime} d^{-1} \in \mathcal{J}$. This implies that $a^{\prime} d^{-1} \in \tilde{\mathcal{J}}$. Now $\tilde{\mathcal{J}}$ has stable rank one, therefore we can pick $f \in \operatorname{inv}(\tilde{\mathcal{T}})=\operatorname{inv}(\mathcal{A}) \cap \tilde{\mathcal{J}}$ such that $\left\|a^{\prime} d^{-1}-f\right\|<\epsilon / 2\|d\|$. Clearly $f d \in \operatorname{inv}(\mathcal{A})$. Also,

$$
\|a-f d\| \leq\left\|a-a^{\prime}\right\|+\left\|a^{\prime}-f d\right\| \leq\left\|a-a^{\prime}\right\|+\left\|a^{\prime} d^{-1}-f\right\|\|d\|<\epsilon,
$$

which shows that $\mathcal{A}$ has indeed stable rank one.

Lemma 5.5 Let $\left(\mathcal{A}_{n}\right)$ be a sequence of unital Banach algebras all of which have stable rank one. Let $\mathcal{J}=c_{0}\left(\mathcal{A}_{n}\right)$, considered as a closed, two-sided ideal in $\mathcal{A}=\ell^{\infty}\left(\mathcal{A}_{n}\right)$. Then $\tilde{\mathcal{J}}$ has stable rank one.

Proof This follows from [25, Theorem 5.2], but we give the argument in this special case. Let $A=\left(t 1_{n}+a_{n}\right) \in \tilde{\mathcal{J}}$, so $\left\|a_{n}\right\| \rightarrow 0$ and $t \in \mathbb{C}$. We wish to approximate $A$ by a member of $\operatorname{inv}(\tilde{\mathcal{J}})$. If $t=0$ then pick $s \in \mathbb{C}$ non-zero and close to $t$. If $A^{\prime}=\left(s 1_{n}+a_{n}\right) \in$ $\tilde{\mathcal{J}}$ can approximated by a member of $\operatorname{inv}(\tilde{\mathcal{J}})$ then so can $A$, because $A^{\prime}$ is close to $A$. So we may suppose that $t \neq 0$. If $t^{-1} A=\left(1_{n}+t^{-1} a_{n}\right)$ can be approximated by a member of $\operatorname{inv}(\tilde{\mathcal{J}})$ then so can $A$.

So we may suppose that $t=1$. Pick $\epsilon>0$ and choose $N$ so that $\left\|a_{n}\right\|<1 / 2$ for $n \geq N$. For $n \geq N$ let $c_{n}=-a_{n}+a_{n}^{2}-a_{n}^{3}+\cdots \in \mathcal{A}_{n}$, hence $\left\|c_{n}\right\| \leq\left\|a_{n}\right\|\left(1-\left\|a_{n}\right\|\right)^{-1}$ and $c_{n} a_{n}=a_{n} c_{n}=-c_{n}-a_{n}$. For $n<N$ use that $\mathcal{A}_{n}$ has stable rank one to find $d_{n} \in \operatorname{inv}\left(\mathcal{A}_{n}\right)$ with $\left\|1_{n}+a_{n}-d_{n}\right\| \leq \epsilon$. Set $c_{n}=\left(d_{n}\right)^{-1}-1_{n}$ for $n<N$. Set $b_{n}=d_{n}-1_{n}$ for $n<N$ and $b_{n}=a_{n}$ for $n \geq N$. Then $B=\left(b_{n}\right), C=\left(c_{n}\right) \in \mathcal{J}$. Notice that

$$
\left(1_{n}+b_{n}\right)\left(1_{n}+c_{n}\right)= \begin{cases}d_{n} d_{n}^{-1} & \text { if } n<N \\ 1_{n}+a_{n}+c_{n}+a_{n} c_{n} & \text { if } n \geq N\end{cases}
$$

and so $\left(1_{n}+b_{n}\right)\left(1_{n}+c_{n}\right)=1_{n}$ for all $n$. Similarly $\left(1_{n}+c_{n}\right)\left(1_{n}+b_{n}\right)=1_{n}$ for all $n$. As $1+B, 1+C \in \tilde{\mathcal{J}}$ we see that $1+B \in \operatorname{inv}(\tilde{\mathcal{J}})$. Finally we consider $\|A-(1+B)\|$. For $n<N$ we have that $\left\|\left(1_{n}+a_{n}\right)-\left(1_{n}+b_{n}\right)\right\|=\left\|1_{n}+a_{n}-d_{n}\right\| \leq \epsilon$, while for $n \geq N$ we have that $\left(1_{n}+a_{n}\right)-\left(1_{n}+b_{n}\right)=a_{n}-a_{n}=0$. Hence $\|A-(1+B)\| \leq \epsilon$.

Proposition 5.6 Let $\left(\mathcal{A}_{n}\right)$ be a sequence of unital Banach algebras all of which have stable rank one. $\ell^{\infty}\left(\mathcal{A}_{n}\right)$ has stable rank one if and only if $\operatorname{Asy}\left(\mathcal{A}_{n}\right)$ has stable rank one.

Proof If $\ell^{\infty}\left(\mathcal{A}_{n}\right)$ has stable rank one then clearly so does $\operatorname{Asy}\left(\mathcal{A}_{n}\right)$. Conversely, set $\mathcal{A}=\ell^{\infty}\left(\mathcal{A}_{n}\right)$ and $\mathcal{J}=c_{0}\left(\mathcal{A}_{n}\right)$ so that $\operatorname{Asy}\left(\mathcal{A}_{n}\right)=\mathcal{A} / \mathcal{J}$. By Lemma 5.5 , we see that $\tilde{\mathcal{J}}$ has stable rank one, and by Lemma 5.1 we know that $\operatorname{inv}(\mathcal{A} / \mathcal{J})=\pi(\operatorname{inv}(\mathcal{A}))$. Thus Proposition 5.4 applies to show that $\mathcal{A}$ has stable rank one. 


\subsection{For $C^{*}$-algebras}

We recall that in a $C^{*}$-algebra $\mathcal{A}$ an element $a \in \mathcal{A}$ has a unitary polar decomposition if there exists a unitary $u \in \mathcal{A}$ such that $a=u|a|$.

Lemma 5.7 If $\mathcal{A}$ is a unital $C^{*}$-algebra such that every element of $\mathcal{A}$ has a unitary polar decomposition then $\mathcal{A}$ has stable rank one.

Proof Let $a \in \mathcal{A}$ and $\epsilon>0$ be fixed. By the assumption there is a unitary $u \in \mathcal{A}$ such that $a=u|a|$. By the Spectral Theorem, we know that $|a|+\epsilon 1 \in \operatorname{inv}(\mathcal{A})$, hence also $b=u(|a|+\epsilon 1) \in \operatorname{inv}(\mathcal{A})$. Then $\|a-b\|=\|\epsilon u\|=\epsilon$. It follows that $\mathcal{A}$ has stable rank one.

Proposition 5.8 Let $\left(\mathcal{A}_{n}\right)$ be a sequence of unital $C^{*}$-algebras having stable rank one. Then $\operatorname{Asy}\left(\mathcal{A}_{n}\right)$, and hence also $\ell^{\infty}\left(\mathcal{A}_{n}\right)$, have stable rank one.

Proof This relies on an observation of Loring, [21, Lemma 19.2.2], which says that under this hypothesis, every element of $\operatorname{Asy}\left(\mathcal{A}_{n}\right)$ has a unitary polar decomposition. The result now follows from Lemma 5.7 and Proposition 5.6.

For completeness, we give the short proof of [21, Lemma 19.2.2]. Let $a \in \operatorname{Asy}\left(\mathcal{A}_{n}\right)$ be $a=\pi(A)$ for some $A=\left(a_{n}\right) \in \ell^{\infty}\left(\mathcal{A}_{n}\right)$. As for each $n$ we have that $\operatorname{inv}\left(\mathcal{A}_{n}\right)$ is dense in $\mathcal{A}_{n}$, we can find $x_{n} \in \operatorname{inv}\left(\mathcal{A}_{n}\right)$ with $\lim _{n \rightarrow \infty}\left\|a_{n}-x_{n}\right\|=0$, so that $a=\pi\left(A^{\prime}\right)$ with $A^{\prime}=\left(x_{n}\right) \in \ell^{\infty}\left(\mathcal{A}_{n}\right)$. Notice that $\left(\left\|x_{n}^{-1}\right\|\right)$ might well be unbounded. For each $n$ set $u_{n}=$ $x_{n}\left(x_{n}^{*} x_{n}\right)^{-1 / 2}$, which is a unitary in $\mathcal{A}_{n}$ with $u_{n}\left|x_{n}\right|=x_{n}$. Then $U=\left(u_{n}\right) \in \ell^{\infty}\left(\mathcal{A}_{n}\right)$ and $X=\left(\left|x_{n}\right|\right) \in \ell^{\infty}\left(\mathcal{A}_{n}\right)$ are such that $U$ is unitary and $X=\left|A^{\prime}\right|$, and $A^{\prime}=U X$. By uniqueness of positive square-roots, $\pi(X)=|a|$ and so $\pi(U)|a|=a$ in $\operatorname{Asy}\left(\mathcal{A}_{n}\right)$ is the required unitary polar decomposition.

Remark 5.9 This result, together with Proposition 5.3, shows that if $\mathcal{A}$ is a $C^{*}$-algebra with stable rank one, then we get a form of uniform norm control on the approximating invertible elements. It would be interesting to know if this could be proved "directly", in some sense.

\subsection{A counter-example}

We shall now present a construction which shows that Proposition 5.8 does not hold for Banach algebras.

Theorem 5.10 The Banach algebra $\mathcal{A}=\ell^{1}(\mathbb{Z})$, equipped with the convolution product, has stable rank one. For any non-principal ultrafilter $\mathcal{U}$ we have that $(\mathcal{A}) \mathcal{U}$ does not have stable rank one, and hence also $\operatorname{Asy}(\mathcal{A})$ and $\ell^{\infty}(\mathcal{A})$ do not have stable rank one.

Proof Let $\left(p_{n}\right)$ be an increasing enumeration of the primes. We shall first show that the ultraproduct $\left(\ell^{1}\left(\mathbb{Z} / p_{n} \mathbb{Z}\right)\right)_{\mathcal{U}}$ does not have stable rank one. We do this by collecting certain facts: 
(1) There is a contractive surjective homomorphism from $\left(\ell^{1}\left(\mathbb{Z} / p_{n} \mathbb{Z}\right)\right) \mathcal{U}$ to $\ell^{1}(G)$ where $G$ is the set-theoretic ultraproduct $\left(\mathbb{Z} / p_{n} \mathbb{Z}\right) \mathcal{U}$. This follows from [7, Section 2.3.2] following [5, Section 5.4]. Notice that $G$ is also a commutative group.

(2) The proof of [20, Theorem 7.1] shows that $G$ is divisible and torsion-free and that $G$ has cardinality the continuum. It follows that there is a set $I$ of continuum cardinality with $G$ and $\oplus_{I} \mathbb{Q}$ isomorphic as $\mathbb{Q}$-vector spaces, so certainly isomorphic as abelian groups. So $\ell^{1}(G)$ is Banach-algebra isomorphic to $\ell^{1}\left(\oplus_{I} \mathbb{Q}\right)$.

(3) Let $H=\oplus_{I} \mathbb{Q}$ and let $\widehat{H}$ be the dual group, a compact abelian group. By [12, Proposition 4.14], for example, we know that the Gel'fand tranform (identified with the Fourier transform) gives a contractive homomorphism $\mathcal{G}: \ell^{1}\left(\oplus_{I} \mathbb{Q}\right) \rightarrow C(\widehat{H})$ which has dense range.

(4) The compact space $\widehat{H}$ consists of all group homomorphisms $\oplus_{I} \mathbb{Q} \rightarrow \mathbb{T}$, equipped with the topology of pointwise convergence. It is easy to see that this agrees with the compact space $(\widehat{\mathbb{Q}})^{I}$. So $C(\widehat{H})$ is isomorphic with $C\left((\widehat{\mathbb{Q}})^{I}\right)$.

(5) There is hence a dense range homomorphism $\left(\ell^{1}\left(\mathbb{Z} / p_{n} \mathbb{Z}\right)\right) \mathcal{U} \rightarrow C\left((\widehat{\mathbb{Q}})^{I}\right)$.

(6) The compact abelian group $\widehat{\mathbb{Q}}$ is identified in [16, Section 25.4]. In particular, it is isomorphic to the " $a$-adic solenoid" $\Sigma_{a}$ for a suitable choice of sequence $a$. These compact groups are studied in [16, Section 10], and in particular, [16, Theorem 10.13] shows that $\Sigma_{a}$ is connected (and compact Hausdorff). It follows from the definition, and [16, Theorem 10.5], that $\Sigma_{a}$ is a metrisable space.

(7) We now consider the covering dimension of a topological space, see for example [23, Chapter 3]. In particular, it follows from [23, Proposition 1.3] that for a Hausdorff space $X$, if $\operatorname{dim}(X)=0$ then $X$ is totally disconnected. Thus $\operatorname{dim}(\widehat{\mathbb{Q}}) \geq 1$. We shall also consider the small inductive dimension of a topological space, [23, Chapter 4]. For a metric space, this is the same as the covering dimension, [23, Section 4, Chapter 5]. Finally, if $X$ is a compact metric space with $\operatorname{dim}(X) \geq 1$, and $I$ an infinite set, then $X^{I}$ has infinite dimension. This is shown for the small inductive dimension in $[8$, Example 1.5.17], and hence also holds for the covering dimension.

(8) Rieffel's original motivation in [25] was to generalise the covering dimension to $C^{*}$ algebras (compare [25, Theorem 1.1] with [23, Proposition 3.3.2] for example). In particular, [25, Proposition 1.7] shows that if $X$ is a compact (Hausdorff) space then the topological stable $\operatorname{rank}$ of $C(X)$ is $\lfloor\operatorname{dim}(X) / 2\rfloor+1$.

(9) In particular, $\widehat{\mathbb{Q}}^{I}$ has infinite dimension. It follows that $C\left(\widehat{\mathbb{Q}}^{I}\right)$ does not have stable rank one. Hence also $\left(\ell^{1}\left(\mathbb{Z} / p_{n} \mathbb{Z}\right)\right)_{\mathcal{U}}$ does not have stable rank one.

As $\ell^{1}(\mathbb{Z}) \rightarrow \ell^{1}\left(\mathbb{Z} / p_{n} \mathbb{Z}\right)$ is a quotient map, for each $n$, it follows that $(\mathcal{A}) \mathcal{U} \rightarrow$ $\left(\ell^{1}\left(\mathbb{Z} / p_{n} \mathbb{Z}\right)\right)_{\mathcal{U}}$ is a quotient map, and so $(\mathcal{A})_{\mathcal{U}}$ does not have stable rank one. As $\operatorname{Asy}(\mathcal{A})$ quotients onto $(\mathcal{A}) \mathcal{U}$, also Asy $(\mathcal{A})$ does not have stable rank one. By Lemma 5.1, also $\ell^{\infty}(\mathcal{A})$ does not have stable rank one.

Finally, we claim that $\mathcal{A}$ does have stable rank one. This follows from the more general result [6, Corollary 1.6]. In fact, using that $\widehat{\mathbb{Z}}=\mathbb{T}$ and that $C(\mathbb{T})$ obviously has a dense set of invertibles, we can instead appeal to [6, Proposition 1.3].

Remark 5.11 The reader may wonder where the argument in the proof of Theorem 5.10 breaks if we attempt to apply it to group $C^{*}$-algebras instead of $\ell^{1}$ group algebras. More concretely, let us consider the $\mathcal{A}:=C^{*}(\mathbb{Z})$, which is isomorphic as a 
$C^{*}$-algebra to $C(\mathbb{T})$. As $\mathbb{Z}$ and $\mathbb{Z} / p_{n} \mathbb{Z}$ are discrete and amenable, the group homomorphism $\mathbb{Z} \rightarrow \mathbb{Z} / p_{n} \mathbb{Z}$ induces a surjective $*$-homomorphism $\mathcal{A} \rightarrow C^{*}\left(\mathbb{Z} / p_{n} \mathbb{Z}\right)$. From [25, Proposition 1.7], we see that $\mathcal{A}$ has stable rank one, and hence so does $C^{*}\left(\mathbb{Z} / p_{n} \mathbb{Z}\right)$. From Proposition 5.8 we know that $\operatorname{Asy}\left(C^{*}\left(\mathbb{Z} / p_{n} \mathbb{Z}\right)\right)$, and thus also $\left(C^{*}\left(\mathbb{Z} / p_{n} \mathbb{Z}\right)\right) \mathcal{U}$, has stable rank one.

On the other hand, when inspecting and adapting the reasoning in the proof of Theorem 5.10, we find that there are *-isomorphisms between $C^{*}(G)$ and $C^{*}\left(\oplus_{I} \mathbb{Q}\right)$ and also with $C\left(\widehat{\mathbb{Q}}^{I}\right)$, where $G$ is the set-theoretic ultraproduct $\left(\mathbb{Z} / p_{n} \mathbb{Z}\right) \mathcal{U}$. As we have just seen, $C\left(\widehat{\mathbb{Q}}^{I}\right)$ does not have stable rank one, hence neither does $C^{*}(G)$. This means that there cannot be a continuous dense range algebra homomorphism from $\left(C^{*}\left(\mathbb{Z} / p_{n} \mathbb{Z}\right)\right) \mathcal{U}$ to $C^{*}(G)$, unlike for $\ell^{1}$ group algebras.

\subsection{For ultraproducts}

We quickly consider what happens when $\operatorname{Asy}\left(\mathcal{A}_{n}\right)$ is replaced by an ultraproduct $\left(\mathcal{A}_{n}\right) \mathcal{U}$. We first adapt Lemma 5.1.

Lemma 5.12 Let $\left(\mathcal{A}_{n}\right)$ be a sequence of unital Banach algebras, let $\mathcal{U}$ be an ultrafilter, and denote by $\pi$ the quotient map $\ell^{\infty}\left(\mathcal{A}_{n}\right) \rightarrow\left(\mathcal{A}_{n}\right)_{\mathcal{U}}$. Then

$$
\operatorname{inv}\left(\left(\mathcal{A}_{n}\right) \mathcal{U}\right)=\pi\left(\operatorname{inv}\left(\ell^{\infty}\left(\mathcal{A}_{n}\right)\right)\right)
$$

Proof We can follow closely the proof of Lemma 5.1. Let $a_{n}, c_{n}, u_{n}, v_{n}$ be as before, and notice now that as $\pi(A) \pi(C)=\pi(C) \pi(A)=1$ there is some set $X \in \mathcal{U}$ with $\left\|u_{n}-1_{n}\right\|<$ $1 / 2$ and $\left\|v_{n}-1_{n}\right\|<1 / 2$ for $n \in X$. Then $a_{n} \in \operatorname{inv}\left(\mathcal{A}_{n}\right)$ and $\left\|a_{n}^{-1}\right\| \leq 2\|C\|$ for each $n \in X$. Hence we can find $A^{\prime} \in \ell^{\infty}\left(\mathcal{A}_{n}\right)$ with $A^{\prime}$ invertible and $\pi(A)=\pi\left(A^{\prime}\right)$.

Proposition 5.2 continues to hold, so if $(\mathcal{A}) \mathcal{U}$ has stable rank one, then so does $\mathcal{A}$. Similarly, a close examination of the proof of Proposition 5.3 shows that it holds also for $(\mathcal{A}) \mathcal{U}$. In particular, we have:

Corollary 5.13 Let $\mathcal{A}$ be a Banach algebra. Then $\operatorname{Asy}(\mathcal{A})$ has stable rank one if and only if $(\mathcal{A})_{\mathcal{U}}$ has stable rank one.

Finally, obviously then Proposition 6.9 shows that if $\left(\mathcal{A}_{n}\right)$ is a sequence of unital $C^{*}$ algebras having stable rank one, then also $\left(\mathcal{A}_{n}\right) \mathcal{U}$ also has stable rank one. Theorem 5.10 shows that this is not true for Banach algebras replacing $C^{*}$-algebras.

\section{Open questions}

We close the paper with some open questions.

- Does the analogue of Proposition 3.16 hold for $C_{\mathrm{DI}}$ ?

- Does the analogue of Proposition 4.15 hold for $C_{\mathrm{PI}}$ ?

- If $\operatorname{Asy}\left(\mathcal{A}_{n}\right)$ has stable rank one, does $\mathcal{A}_{n}$ for large enough $n$ ?

- Can one find a counter-example as in Theorem 5.10 which uses directly the criteria established in Proposition 5.3? 
A wider "open question" is to study the constants $C_{\mathrm{DI}}$ and $C_{\mathrm{PI}}$, and the criteria from Proposition 5.3. These are "metric versions" of being Dedekind-infinite, being properly infinite, and having stable rank one. We wonder if there are other properties of Banach algebras which have interesting "metric versions"?

Acknowledgement The authors are grateful to Yemon Choi (Lancaster), James Gabe (Copenhagen), Saeed Ghasemi (Prague), Tomasz Kania (Prague) and Jared White (London) for many enlightening conversations. The authors are indebted to Niels J. Laustsen (Lancaster) for drawing their attention to the paper [4]. We are extremely grateful to the anonymous referee whose comments much improved the exposition of the paper. The second-named author acknowledges with thanks the funding received from GAČR project 19-07129Y; RVO 67985840 (Czech Republic).

\section{References}

[1] I. Ben Yaacov, A. Berenstein, C. W. Henson and A. Usvyatsov, Model theory for metric structures, in Model theory with applications to algebra and analysis. Vol. 2, 315-427, London Math. Soc. Lecture Note Ser., 350, Cambridge Univ. Press, Cambridge. MR2436146

[2] B. Blackadar, $K$-theory for operator algebras, second edition, Mathematical Sciences Research Institute Publications, 5, Cambridge University Press, Cambridge, 1998. MR1656031

[3] H. G. Dales, Banach algebras and automatic continuity, London Mathematical Society Monographs. New Series, 24, The Clarendon Press, Oxford University Press, New York, 2000. MR1816726

[4] H. G. Dales, N. J. Laustsen and C. J. Read, A properly infinite Banach *-algebra with a non-zero, bounded trace, Studia Math. 155 (2003), no. 2, 107-129. MR1961188

[5] M. Daws, Amenability of ultrapowers of Banach algebras, Proc. Edinb. Math. Soc. (2) 52 (2009), no. 2, 307-338. MR2506395

[6] T. W. Dawson and J. F. Feinstein, On the denseness of the invertible group in Banach algebras, Proc. Amer. Math. Soc. 131 (2003), no. 9, 2831-2839. MR1974340

[7] S. Draga and T. Kania, When is multiplication in a Banach algebra open?, Linear Algebra Appl. 538 (2018), 149-165. MR3722833

[8] R. Engelking, Dimension theory, translated from the Polish and revised by the author, North-Holland Publishing Co., Amsterdam, 1978. MR0482697

[9] I. Farah, Combinatorial set theory of $C^{*}$-algebras, Springer Monographs in Mathematics, Springer, 2019

[10] I. Farah, B. Hart, M. Lupini, L. Robert, A. Tikuisis, A. Vignati, and W. Winter, Model theory of $C^{*}-$ algebras, to appear in Memoirs of the Amer. Math. Soc, see arXiv:1602.08072 [math. LO]

[11] I. Farah, B. Hart and D. Sherman, Model theory of operator algebras II: model theory, Israel J. Math. 201 (2014), no. 1, 477-505. MR3265292

[12] G. B. Folland, A course in abstract harmonic analysis, second edition, Textbooks in Mathematics, CRC Press, Boca Raton, FL, 2016. MR3444405

[13] P. Friis and M. Rørdam, Almost commuting self-adjoint matrices-a short proof of Huaxin Lin's theorem, J. Reine Angew. Math. 479 (1996), 121-131. MR1414391

[14] S. Ghasemi, Reduced products of metric structures: a metric Feferman-Vaught theorem, J. Symb. Log. 81 (2016), no. 3, 856-875. MR3569108

[15] S. Heinrich, Ultraproducts in Banach space theory, J. Reine Angew. Math. 313 (1980), 72-104. MR0552464

[16] E. Hewitt and K. A. Ross, Abstract harmonic analysis. Vol. I, second edition, Grundlehren der Mathematischen Wissenschaften, 115, Springer-Verlag, Berlin, 1979. MR0551496

[17] B. Horváth, Banach spaces whose algebras of operators are Dedekind-finite but they do not have stable rank one, (accepted in Proc. 24th International Conference on Banach Algebras and Applications (editor: M. Filali)). arXiv: 1807.10578

[18] B. E. Johnson, Approximately multiplicative maps between Banach algebras, J. London Math. Soc. (2) 37 (1988), no. 2, 294-316. MR0928525 
[19] E. Kirchberg, Central sequences in $C^{*}$-algebras and strongly purely infinite algebras, in Operator Algebras: The Abel Symposium 2004, 175-231, Abel Symp., 1, Springer, Berlin. MR2265050

[20] S. Kochen, Ultraproducts in the theory of models, Ann. of Math. (2) 74 (1961), 221-261. MR0138548

[21] T. A. Loring, Lifting solutions to perturbing problems in $C^{*}$-algebras, Fields Institute Monographs, 8, American Mathematical Society, Providence, RI, 1997. MR1420863

[22] T. W. Palmer, Banach algebras and the general theory of *-algebras. Vol. I, Encyclopedia of Mathematics and its Applications, 49, Cambridge University Press, Cambridge, 1994. MR1270014

[23] A. R. Pears, Dimension theory of general spaces, Cambridge University Press, Cambridge, England, 1975. MR0394604

[24] J. Renault, A groupoid approach to $C^{*}$-algebras, Lecture Notes in Mathematics, 793, Springer, Berlin, 1980. MR0584266

[25] M. A. Rieffel, Dimension and stable rank in the $K$-theory of $C^{*}$-algebras, Proc. London Math. Soc. (3) 46 (1983), no. 2, 301-333. MR0693043

[26] M. Rørdam, F. Larsen and N. Laustsen, An introduction to $K$-theory for $C^{*}$-algebras, London Mathematical Society Student Texts, 49, Cambridge University Press, Cambridge, 2000. MR1783408

[27] M. Takesaki, Theory of operator algebras. III, Encyclopaedia of Mathematical Sciences, 127, SpringerVerlag, Berlin, 2003. MR1943007

[28] J. Zemánek, Idempotents in Banach algebras, Bull. London Math. Soc. 11 (1979), no. 2, 177-183. MR0541972

Jeremiah Horrocks Institute, University of Central Lancashire, Preston, PR1 2HE, United Kingdom

e-mail:mdaws@uclan.ac.uk.

Institute of Mathematics of the Czech Academy of Sciences, Prague, Czech Republic

e-mail: horvath@math.cas.cz. 